\title{
Selections and suborderability
}

\author{
by
Giuliano Artico (Padova), Umberto Marconi (Padova), Jan Pelant (Praha), Luca Rotter (Padova) and Mikhail Tkachenko (México, D.F.)

\begin{abstract}
We extend van Mill-Wattel's results and show that each countably compact completely regular space with a continuous selection on couples is suborderable. The result extends also to pseudocompact spaces if they are either scattered, first countable, or connected. An infinite pseudocompact topological group with such a continuous selection is homeomorphic to the Cantor set. A zero-selection is a selection on the hyperspace of closed sets which chooses always an isolated point of a set. Extending Fujii-Nogura results, we show that an almost compact space with a continuous zero-selection is homeomorphic to some ordinal space, and a (locally compact) pseudocompact space with a continuous zero-selection is an (open) subspace of some space of ordinals. Under the Diamond Principle, we construct several counterexamples, e.g. a locally compact locally countable monotonically normal space with a continuous zero-selection which is not suborderable.
\end{abstract}

0. Introduction. Throughout this paper, all spaces are assumed to be Hausdorff. Let $\mathfrak{F}(X)$ be the set of all non-empty closed subsets of $X$. A selection for $X$ is a map $\varphi: \mathfrak{F}(X) \rightarrow X$ such that $\varphi(F) \in F$ for all $F \in \mathfrak{F}(X)$. Denote by $[X]^{2}$ the subset of $\mathfrak{F}(X)$ consisting of the subsets with exactly two elements (couples). A weak selection on $X$ is a map $\sigma:[X]^{2} \rightarrow X$ such that $\sigma(\{x, y\}) \in\{x, y\}$ for all couples.

For every subset $V$ of $X$, let $V^{+}=\{F \in \mathfrak{F}(X): F \subseteq V\}$ and $V^{-}=$ $\{F \in \mathfrak{F}(X): F \cap V \neq \emptyset\}$. The Vietoris topology on $\mathfrak{F}(X)$ has a base consisting of the sets of the form $V^{+} \cap W_{1}^{-} \cap \ldots \cap W_{n}^{-}$, where $V, W_{1}, \ldots, W_{n}$ are open subsets of $X$.

2000 Mathematics Subject Classification: 54C65, 54B20, 54F05, 54H11, 54G12.

Key words and phrases: (weak) selection, countably compact, pseudocompact, (sub)orderable, topological group, scattered space, Vietoris topology, Fell topology, ordinal space.

Work supported by the research project "Metodi e problemi in Analisi Reale" of the Italian Ministero dell'Università e della Ricerca Scientifica e Tecnologica and the grant GA ČR 201/00/1466. 
By a continuous selection (resp., continuous weak selection) on $X$, we mean a selection (resp., weak selection) which is continuous for the Vietoris topology on $\mathfrak{F}(X)$ (resp., on the subspace $[X]^{2}$ ). In Section 2, when we consider the continuity of selections with respect to the Fell topology, this difference is mentioned explicitly.

If $\sigma$ is a continuous weak selection, it will be convenient sometimes to consider the corresponding continuous map $\sigma_{0}: X \times X \rightarrow X$ defined by $\sigma_{0}(x, y)=\sigma(\{x, y\})$ for distinct $x, y \in X$ and $\sigma_{0}(x, x)=x$ for each $x \in X$. Conversely, every continuous map $\sigma_{0}: X \times X \rightarrow X$ satisfying $\sigma_{0}(x, y)=\sigma_{0}(y, x) \in\{x, y\}$ and $\sigma_{0}(x, x)=x$ for all $x, y \in X$ determines a continuous weak selection $\sigma:[X]^{2} \rightarrow X$. Therefore, in what follows we do not distinguish between $\sigma$ and $\sigma_{0}$. Notice that a weak selection is continuous if and only if it locally coincides with the projection of $X \times X$ to the first or the second factor, at any point $(x, y)$ with $x \neq y$.

In [29], van Mill and Wattel proved that a compact space $X$ has a weak selection iff it is orderable, i.e., the topology of $X$ coincides with the open interval topology of a linear order on $X$. A topological space is said to be suborderable provided that it is a subspace of an orderable space. It is well known that a space $X$ is suborderable if and only if it is a GO-space, that is, there exists a base for $X$ consisting of subsets which are convex for a suitable linear order on $X$ [6, Theorems 17 A.22, 17 A.23].

A useful characterization of suborderable spaces by means of continuous weak selections is given in [30]. We use it in Section 1 to present a criterion of suborderability for pseudocompact spaces which generalizes the compact case in [29]. As a corollary, we show that a countably compact Tikhonov space with a continuous weak selection is suborderable. Furthermore, a pseudocompact space with a continuous weak selection is suborderable if it is either scattered, first countable, or connected.

The existence of a continuous weak selection on topological groups has a strong impact on the topological properties of the groups. By Theorem 1.26, a locally pseudocompact group with a continuous weak selection is either discrete or has an open neighborhood of the identity homeomorphic to the Cantor set or to the reals $\mathbb{R}$. In particular, a pseudocompact group with a continuous weak selection is either finite or topologically homeomorphic to the Cantor set (Corollary 1.28).

A selection $\varphi$ on a space $X$ is said to be a zero-selection if $\varphi(F)$ is an isolated point of $F$ for each $F \in \mathfrak{F}(X)$. By a continuous zero-selection we mean a zero-selection which is continuous for the Vietoris topology. Obviously, a space with a zero-selection is scattered and a continuous zeroselection for $X$ induces a continuous zero-selection for every closed subspace of $X$ (in fact, for every subspace if $X$ is normal, see Proposition 2.10). 
Fujii and Nogura [14] proved that a compact space is homeomorphic to an ordinal space iff it has a continuous zero-selection (an ordinal space is an ordinal equipped with the order topology). They raised a question whether it is possible to characterize ordinal spaces in locally compact spaces by means of continuous selections under the condition of local compactness.

In Sections 2 and 3, we obtain several affirmative results in this direction; however, two counterexamples are given in Section 4. We prove in Theorem 2.5 that the existence of a Fell-continuous zero-selection on $X$ ensures that the space $X$ is homeomorphic to an ordinal space (the existence of a Fell-continuous selection on $[X] \leq 2$ implies that $X$ is locally compact and the one-point compactification of $X$ is ordered; see [3] and [20]). As the Fell and Vietoris topologies coincide for compact spaces, Theorem 2.5 generalizes Fujii-Nogura's result from [14]. Theorem 2.7 provides another generalization: an almost compact space with a continuous zero-selection is proven to be homeomorphic to an ordinal space. The main result of Section 2 is Theorem 2.9 where we prove that if $X$ is a (locally compact) pseudocompact space with a continuous zero-selection, then $X$ is homeomorphic to an (open) subspace of an ordinal space.

If $X$ is a locally compact paracompact space with a continuous zeroselection, then $X$ can be embedded as an open subspace into an ordinal space (Theorem 3.1). Furthermore, if $X$ is also Lindelöf, then it is homeomorphic to an ordinal space of countable cofinality $\left({ }^{1}\right)$.

One may ask (cf. [14]) whether the existence of a continuous zero-selection on a locally compact space $X$ implies the existence of an embedding of $X$ into an ordinal space. In Section 3, we discuss this question for locally compact locally countable spaces (LCC-spaces for short). Affirmative results of Section 3 provide "bounds" for possible counterexamples. In particular, we prove in Theorem 3.4 (resp., Theorem 3.5) that if the Cantor-Bendixson height of an LCC-space $X$ is countable (resp., finite), then $X$ is an open subspace of an ordinal space, provided that $X$ is hereditarily collectionwise normal (resp., collectionwise normal). Furthermore, the condition on the height of $X$ cannot be removed, even if $X$ has a continuous zero-selection. Under the Diamond Principle, we provide several counterexamples in Section 4. In Theorem 4.6, we construct a monotonically normal LCC-space with a continuous zero-selection which is not suborderable. In Theorem 4.7, we show that a collectionwise normal LCC-space of countable height may have a continuous zero-selection without being suborderable. Moreover, an LCC-space with a continuous zero-selection is not necessarily normal, even if the Cantor-Bendixson height is equal to 2. Finally, we discuss some open problems in Section 5.

$\left({ }^{1}\right)$ For the compact case, notice that the cofinality of a successor ordinal is equal to 1. 


\section{Weak selections}

1.1. Extending van Mill-Wattel's results. By [29, Theorem 1.1], a compact Hausdorff space with a continuous weak selection is linearly orderable. We are going to generalize this result to wider classes of spaces, including all countably compact Tikhonov spaces as well as the spaces with the pseudocompact square. On this way, we use and develop some ideas from [30]. At the same time, we hope that one can go further extending our results (see Problem 5.2).

In [30], the following property of a selection $\sigma:[X]^{2} \rightarrow X$ was introduced:

Definition 1.1. A continuous weak selection $\sigma$ on $X$ is called locally uniform provided that for every $x \in X$ and for every neighborhood $U$ of $x$, there is a neighborhood $V$ of $x$ which is contained in $U$ and such that for all $p \in X \backslash U$ and $y \in V$,

$$
\sigma(p, y)=p \quad \text { iff } \quad \sigma(p, x)=p .
$$

It is proved in [30] that every suborderable space admits a locally uniform weak selection and each continuous weak selection on a compact space is locally uniform (cf. Lemma 1.3 below). The following result from [30] shows the importance of locally uniform weak selections.

THEOREM 1.2. The following conditions are equivalent for every completely regular space $X$ :

(1) $X$ has a locally uniform weak selection;

(2) $X$ is suborderable.

Let us prove the following lemma which via Theorem 1.2 gives the promised extension of [29, Theorem 1.1] to the spaces whose square is pseudocompact. As usual, pseudocompact spaces are assumed to be completely regular.

Lemma 1.3. Let $X$ be a space such that $X \times X$ is pseudocompact and let $\sigma$ be a continuous weak selection on $X$. Then $\sigma$ is locally uniform.

Proof. Suppose to the contrary that the weak selection $\sigma$ on $X$ is not locally uniform. Then we can find $x \in X$ and a neighborhood $U$ of $x$ in $X$ such that for each smaller neighborhood $V$ of $x$, there are $p_{V} \in X \backslash U$ and $y_{V} \in V$ such that

$$
\begin{aligned}
& \left(\sigma\left(p_{V}, y_{V}\right)=p_{V} \& \sigma\left(p_{V}, x\right)=x\right) \quad \text { or } \\
& \left(\sigma\left(p_{V}, y_{V}\right)=y_{V} \& \sigma\left(p_{V}, x\right)=p_{V}\right) .
\end{aligned}
$$

Now an inductive construction of length $\omega$ follows. For each $n \in \omega$, we find a neighborhood $V_{n}$ of $x$, points $p_{V_{n}}, y_{V_{n}}$, and an open set $\widetilde{G}_{n}$ in $X$ satisfying the following conditions for all $n \in \omega$ : 
(1) $y_{V_{n}} \in V_{n}, p_{V_{n}} \in X \backslash U, p_{V_{n}} \in \widetilde{G}_{n}$;

(2) $(*)$ is satisfied for points $x, y_{V_{n}}, p_{V_{n}}$;

(3) $\bar{V}_{0} \subset U$ and $\bar{V}_{n+1} \subset V_{n}$;

(4) $V_{n+1}$ and $\widetilde{G}_{n}$ witness the continuity of $\sigma$ at $\left(x, p_{V_{n}}\right)$, i.e., $\sigma(w, z)=w$ iff $\sigma\left(p_{V_{n}}, x\right)=p_{V_{n}}$ for each $w \in \widetilde{G}_{n}$ and each $z \in V_{n+1}$.

Without loss of generality we may assume that the set $\left\{p_{V_{n}}: n \in \omega\right\}$ is discrete in itself and that

$$
\sigma\left(p_{V_{n}}, x\right)=x \text {, hence } \sigma\left(p_{V_{n}}, y_{V_{n}}\right)=p_{V_{n}}
$$

for each $n \in \omega$. For every $n \in \omega$, choose open sets $G_{n} \ni p_{V_{n}}$ and $H_{n} \ni y_{V_{n}}$ satisfying

(i) $\left\{G_{n}: n \in \omega\right\} \cup\left\{H_{n}: n \in \omega\right\}$ is a pairwise disjoint family;

(ii) $\bar{H}_{n} \subset V_{n}, G_{n} \subset \widetilde{G}_{n}$ and $\bar{G}_{n} \subset X \backslash \bar{V}_{0}$ for each $n \in \omega$;

(iii) $G_{n}$ and $H_{n}$ witness the continuity of $\sigma$ at $\left(p_{V_{n}}, y_{V_{n}}\right)$, i.e., for any ordered couple $(a, b) \in G_{n} \times H_{n}, \sigma(a, b)=a$ (see $\left.(* *)\right)$.

Consider the family $\mathcal{U}=\left\{G_{n} \times H_{n}: n \in \omega\right\}$ of open sets in $X \times X$. By pseudocompactness of $X \times X$, there is a point $(u, v) \in X \times X$ such that any neighborhood of $(u, v)$ intersects infinitely many elements of $\mathcal{U}$. Then $u \neq v$ by the choice of the $G_{n}$ 's and $H_{n}$ 's. As $\sigma$ is continuous, (iii) implies that $\sigma(u, v)=u$. The continuity of $\sigma$ implies that there exists a neighborhood $W$ of $u$ such that $v \notin W$ and $\sigma(w, v)=w$ for each $w \in W$. On the other hand, $v \in \bigcap\left\{V_{n}: n \in \omega\right\}$. From the choice of $V_{n+1}$ 's and $\widetilde{G}_{n}$ 's it follows that $\sigma(v, w)=v$ for all $w \in G_{n}$ and each $n \in \omega$. We pick $w \in G_{m} \cap W$ for some $m \in \omega$. As $v \notin G_{m} \cap W$, the behavior of $\sigma$ just described yields a contradiction.

Remark 1.3.1. Another way to prove Lemma 1.3 is to apply Glicksberg's theorem [16] to extend $\sigma$ from $X$ to a continuous weak selection $\widetilde{\sigma}$ on the Stone-Cech compactification $\beta X$ and then use the fact that a continuous weak selection on a compact space is locally uniform [30, Lemma 1.1].

Lemma 1.3 has several applications. The following result generalizes [29, Theorem 1.1]. Notice that a countably compact suborderable space is sequentially compact $[41,5.5]$.

Corollary 1.4. Let $X$ be a space with a continuous weak selection. If $X^{2}$ is pseudocompact, then $X$ is sequentially compact and suborderable.

Proof. Lemma 1.3 and Theorem 1.2 together imply that $X$ is suborderable. Since every suborderable space is normal, we conclude that the pseudocompact space $X$ is countably compact, hence sequentially compact.

As the square of a pseudocompact $k$-space is pseudocompact [12, 3.10.26], Corollary 1.4 immediately gives: 
Corollary 1.5. If a pseudocompact $k$-space has a continuous weak selection, then it is sequentially compact and suborderable.

We do not know whether pseudocompactness of $X^{2}$ in Corollary 1.4 can be weakened to pseudocompactness of $X$ (see Problems 5.1 and 5.2 below). However, there are several classes of topological spaces in which pseudocompactness turns out to be stable under the product operation. A good example of this phenomenon is the Comfort-Ross theorem [7] which states that pseudocompactness is productive in the class of topological groups. We shall see in Theorem 1.27 below that the existence of a continuous weak selection on $[X]^{2}$ simplifies a lot the structure of $X$ when $X$ is a (locally) pseudocompact topological group. Another instance of a similar phenomenon is the case of a countably compact Tikhonov space $X$ with a continuous weak selection. The assumption of pseudocompactness of $X^{2}$ in Lemma 1.3 is satisfied automatically since a countably compact space $X$ with a continuous weak selection is sequentially compact (see [9]) and, hence, so is $X \times X$.

Corollary 1.6. A countably compact Tikhonov space with a continuous weak selection is sequentially compact and suborderable.

In fact, the above corollary remains valid for spaces $X$ that contain a dense set all countably infinite subsets of which have accumulation points in $X$. Such spaces are called countably protocompact [2]. Clearly, countably compact spaces are countably protocompact and countably protocompact Tikhonov spaces are pseudocompact, but these implications are not invertible in general. The following result generalizes Corollary 1.6.

Proposition 1.7. A countably protocompact Tikhonov space with a continuous weak selection is sequentially compact and suborderable.

Proof. Let $X$ be a countably protocompact space with a continuous weak selection. Denote by $D$ a dense set in $X$ which witnesses countable protocompactness of $X$. One can follow van Douwen's reasoning from [9] to prove that every infinite subset of $D$ contains a non-trivial sequence converging in $X$. We can say that $D$ is relatively sequentially compact in $X$. Observe that $D \times D$ is relatively sequentially compact in $X \times X$, which implies that $X \times X$ must be pseudocompact. The conclusion now follows from Corollary 1.4.

A simple verification shows that $X \times X$ is still pseudocompact for a pseudocompact scattered space $X$ (see Proposition 1.13). Of course, this does not require any assumption on the existence of a continuous weak selection on $X$. We guess this observation is well known though we have not found it in the literature so far. So, every scattered pseudocompact space with a continuous weak selection is suborderable, hence normal and countably compact. This is one more reason why the Tikhonov plank, the Isbell-Mrówka 
space $\Psi$ and similar spaces have no continuous weak selections. We shall see in Section 4 that under $\diamond$, there is a locally compact locally countable space $X$ with a continuous zero-selection which is not normal though its Cantor-Bendixson height is 2 .

A very important class $\mathcal{B}$ of completely regular spaces was defined by Z. Frolík in [13]. By definition, a space $X$ is in $\mathcal{B}$ if the product $X \times Y$ is pseudocompact for every pseudocompact space $Y$. It is easy to see that $\mathcal{B}$ is a proper subclass of the class of all pseudocompact spaces. The spaces $X \in \mathcal{B}$ admit the following nice characterization $[13,5,42]$ : for every sequence $\left\{U_{n}: n \in \omega\right\}$ of disjoint non-empty open subsets of $X$, there is an infinite subset $J \subseteq \omega$ such that for any filter $\xi$ on $J$, the set $\bigcap_{F \in \xi} \overline{\bigcup_{n \in F} U_{n}}$ is not empty. By [38, Corollary 2.14], every pseudocompact topological group is in $\mathcal{B}$. In addition, from [38, Theorem 2.13] it follows that a dense pseudocompact subspace of a product of compact metrizable spaces belongs to $\mathcal{B}$.

We now define sequentially pseudocompact spaces which form a proper subclass of $\mathcal{B}$.

Definition 1.8. A completely regular space $X$ is called sequentially pseudocompact if for any family $\left\{U_{n}: n \in \omega\right\}$ of pairwise disjoint non-empty open sets, there are an infinite set $J \subseteq \omega$ and a point $x \in X$ such that every neighborhood of $x$ intersects all but finitely many elements of $\left\{U_{n}: n \in J\right\}$.

From the above characterization of the spaces in $\mathcal{B}$ and Definition 1.8 it immediately follows that every sequentially pseudocompact space is in $\mathcal{B}$ :

Proposition 1.9. Every sequentially pseudocompact space belongs to Frolik's class $\mathcal{B}$. Therefore, the product of two sequentially pseudocompact spaces is pseudocompact.

It is worth noting that even compact spaces need not be sequentially pseudocompact: $\beta \omega$ is a counterexample. Since every compact space belongs to $\mathcal{B}$, there are many spaces in $\mathcal{B}$ which fail to be sequentially pseudocompact.

By [38, Corollary 2.14], every pseudocompact group is in $\mathcal{B}$. It turns out that this result admits the following strengthening.

Proposition 1.10. Every pseudocompact topological group $G$ is sequentially pseudocompact.

Proof. Denote by $K$ the completion of $G$. Then $K$ is a compact topological group. By Kuz'minov's theorem [26, 40], $K$ is dyadic. Therefore, there exists a continuous onto map $f: D^{\tau} \rightarrow K$, where $D=\{0,1\}$ is the discrete two-point space and $\tau$ is an appropriate infinite cardinal. Consider a pairwise disjoint family $\left\{U_{n}: n \in \omega\right\}$ of non-empty open sets 
in $G$. Since $G$ is pseudocompact, the family $\left\{U_{n}: n \in \omega\right\}$ has an accumulation point $x \in G$. For every $n \in \omega$, choose an open set $V_{n}$ in $K$ such that $V_{n} \cap G=U_{n}$. The set $W_{n}=f^{-1}\left(V_{n}\right)$ is open in $D^{\tau}$ and its closure depends on at most countably many coordinates [12, 2.7.12]. In other words, for every $n \in \omega$ there exists a countable subset $A_{n}$ of $\tau$ such that $\bar{W}_{n}=\pi_{A_{n}}^{-1}\left(\pi_{A_{n}}\left(\bar{W}_{n}\right)\right)$, where $\pi_{A_{n}}: D^{\tau} \rightarrow D^{A_{n}}$ is the projection. Set $A=\bigcup_{n \in \omega} A_{n}$. Then $\bar{W}_{n}=\pi_{A}^{-1}\left(\pi_{A}\left(\bar{W}_{n}\right)\right)$ for each $n \in \omega$.

Since the map $f$ is closed and $x$ is in the closure of the set $\bigcup_{k \geq n} V_{k}$, we have $f^{-1}(x) \cap \overline{\bigcup_{k=n}^{\infty} W_{n}} \neq \emptyset$ for each $n \in \omega$. Therefore, the set

$$
f^{-1}(x) \cap \bigcap_{n \in \omega} \overline{\bigcup_{k \geq n} W_{k}}
$$

is non-empty. Pick an arbitrary point $y$ from this set and put $z=\pi_{A}(y)$. Clearly, $D^{A}$ is a metrizable compact space and $z$ is an accumulation point of the family $\left\{\pi_{A}\left(W_{n}\right): n \in \omega\right\}$. Therefore, we can find an infinite set $J \subseteq \omega$ such that every neighborhood of $z$ in $D^{A}$ intersects almost all elements of the family $\left\{\pi_{A}\left(W_{n}\right): n \in J\right\}$. Since the regular closed sets $\bar{W}_{n}$ depend only on $A$, we infer that every neighborhood of $y$ in $D^{\tau}$ intersects almost all elements of the family $\left\{W_{n}: n \in J\right\}$. This immediately implies that $x=f(y)$ has the same property with respect to the families $\left\{V_{n}: n \in J\right\}$ and $\left\{U_{n}: n \in J\right\}$ because each $U_{n}$ is dense in $V_{n}$. So, $G$ is sequentially pseudocompact.

A topological space $X$ is said to be scattered if every non-empty subset $Y$ of $X$ has a point relatively isolated in $Y$. The following fact is almost evident.

Proposition 1.11. All pseudocompact scattered spaces and all first countable pseudocompact spaces are sequentially pseudocompact.

Proposition 1.11 admits a generalization given below. First, we need a definition.

Definition 1.12. A topological space $X$ is called $\psi$ - $\omega$-scattered provided that every non-empty closed subset $F$ of $X$ contains a point of countable pseudocharacter in $F$.

Proposition 1.13. Let $X$ be a $\psi$ - $\omega$-scattered pseudocompact space. Then $X$ is sequentially pseudocompact.

Proof. Consider a family $\mathcal{U}=\left\{U_{n}: n \in \omega\right\}$ of pairwise disjoint nonempty open subsets of $X$. Put $F=\{y \in X$ : each neighborhood of $y$ intersects infinitely many elements of $\mathcal{U}\}$. Clearly, $F$ is a non-empty closed set. Therefore, $F$ contains a point $x$ which has countable pseudocharacter in $F$. Take a decreasing collection of open sets $\mathcal{V}=\left\{V_{n}: n \in \omega\right\}$ in $X$ containing $x$ such that $\bigcap\left\{\bar{V}_{n} \cap F: n \in \omega\right\}=\{x\}$. For every $n \in \omega$, choose 
$k_{n}>n$ such that $V_{n} \cap U_{k_{n}} \neq \emptyset$. Put $J=\left\{k_{n}: n \in \omega\right\}$. Assume that there is a neighborhood $W$ of $x$ missing $U_{k_{n}}$ 's for infinitely many members of $J$. Put $K=\left\{k_{n} \in J: W \cap U_{k_{n}}=\emptyset\right\}$. As $K$ is infinite by our assumption, there is a point $q \in F, q \neq x$, such that each neighborhood of $q$ intersects infinitely many elements of the family $\left\{V_{n} \cap U_{k_{n}}: k_{n} \in K\right\}$. But then $q \in \bar{V}_{n}$ for each $n \in \omega$. This contradiction concludes the proof.

COROLlary 1.14. Suppose that $X$ is a sequentially pseudocompact space with a continuous weak selection. Then $X$ is sequentially compact and suborderable.

Proof. The conclusion follows from Corollary 1.4 and Proposition 1.9.

COROLlary 1.15. If $X$ is a pseudocompact $\psi$ - $\omega$-scattered space with a continuous weak selection, then $X$ is sequentially compact and suborderable.

Proof. Apply Proposition 1.13 and Corollary 1.14.

Making use of Corollary 1.15 and Glicksberg's theorem [16], we characterize the orderability of the Cech-Stone compactification $\beta X$ of $X$ as follows (see also [43, 33]):

THEOREM 1.16. Let $X$ be a completely regular space. The following conditions are equivalent:

(i) $\beta X$ is orderable;

(ii) $X$ is suborderable and pseudocompact;

(iii) $X$ is countably compact and has a continuous weak selection;

(iv) $X \times X$ is pseudocompact and $X$ has a continuous weak selection.

Proof. (i) $\Rightarrow$ (ii). Pseudocompactness of $X$ follows from [43, Proposition 3.2].

(ii) $\Rightarrow$ (iii). Every suborderable space is normal and has a continuous weak selection.

(iii) $\Rightarrow$ (iv). Apply Corollary 1.6.

(iv) $\Rightarrow$ (i). A continuous weak selection $\sigma: X \times X \rightarrow X$ can be extended over $\beta(X \times X)=\beta X \times \beta X$ (see also Remark 1.3.1). By the density of $X \times X$ in $\beta X \times \beta X$, the extension is still a continuous weak selection and, consequently, $\beta X$ is orderable [29].

Corollaries 1.6 and 1.15 extend the results of van Mill and Wattel on orderability of compact spaces [29]. Note that a suborderable space is orderable when it is scattered [34] or connected (in the latter case, the subspace topology on a connected subset of a linearly ordered space coincides with the one induced by the linear order).

There are countably compact locally connected suborderable spaces that are not orderable. Let $\left[0, \omega_{1}\right)^{*}$ denote the usual ordinal space $\left[0, \omega_{1}\right)$ with its order reversed, and let $D=\left[0, \omega_{1}\right)^{*} \oplus\left[0, \omega_{1}\right)$. Then let $L$ be the lexicographic 
product $D \times[0,1)$ (this space is sometimes called the "mirrored long line"). Finally, let $X$ be the topological sum of $L$ with a single point $p \notin L$. The resulting space is the required example which shows that van Mill-Wattel's result on orderability cannot be extended outside the realm of compact spaces.

1.2. Weak orderability. A space $X$ is said to be weakly orderable if there exists a coarser orderable topology on $X$; a suitable order on $X$ inducing this coarser open interval topology is called a compatible order for $X$. Suborderable spaces are weakly orderable. If $X$ is weakly orderable, then $\sigma(x, y)=\min \{x, y\}$ is a continuous weak selection (cf. Lemma 2.2 and Problem 5.4 below). Putting together results from [28] and [10], one gets the following:

THEOREM 1.17. A connected space has a continuous weak selection if and only if it is weakly orderable. In this case, there are only two compatible orders (continuous weak selections), each the reverse of the other.

REMARK 1.17.1. The problem of whether each space with a continuous weak selection is weakly orderable was suggested by van Mill and Wattel in [30]; cf. Problem 5.4 below.

One can easily see that a locally connected weakly orderable space is a GO-space and that every connected GO-space is orderable. Consequently, a weakly orderable locally connected and connected space is orderable [10]. Since a locally connected weakly orderable space is locally compact, one may wonder what is the role of local compactness here. It turns out that local compactness and local connectedness are equivalent for connected weakly orderable spaces. Though the idea of our proof goes back to [10], we have not been able to find anywhere an explicit formulation of the following fact.

Proposition 1.18. Let $X$ be a connected weakly orderable space. If $X$ is locally compact, then it is orderable and locally connected.

Proof. Fix a compatible linear order $\leq$ on $X$ and consider the order topology $\tau$ of $(X, \leq)$. The identity map $f$ of $X$ onto the orderable space $(X, \tau)$ is continuous. As shown in [10], $\tau$ is connected and locally connected for any connected weakly orderable space $X$ (such an order is called continuous in [10]). At this moment, one can establish quite a tight connection between $X$ and $\leq$. It turns out that for any $p \in X$, both sets $(\leftarrow, p]$ and $[p, \rightarrow)$ are connected as subsets of $X$. Indeed, suppose that $(\leftarrow, p]=A \cup B$, where $A$ and $B$ are disjoint non-empty and relatively open in $(\leftarrow, p]$ (in the induced topology); without loss of generality, we assume that $p \in A$. Then, clearly, $\{A \cup[p, \rightarrow), B\}$ is a partition of $X$ into non-empty open sets, which is a contradiction. 
We show that the local compactness of $X$ implies that $f: X \rightarrow(X, \tau)$ is open, so $X$ is also orderable and locally connected. Arguing by contradiction, suppose that there exist a point $p \in X$ and a compact neighborhood $K$ of $p$ containing no open interval around $p$. Assume that $p$ is the minimum of $X$ (in this case, an open interval containing $p$ is of the form $[p, q)=(\leftarrow, q)$ ). We have $\inf (X \backslash K)=p$. For every $b \notin K$, the set $K \cap[p, b]=K \cap[p, b)$ is closed and the set $\stackrel{\circ}{K} \cap[p, b]=\stackrel{\circ}{K} \cap[p, b)$ is open ( $K^{\circ}$ denotes the interior of $K)$. Consequently, the set $S_{b}=(K \backslash \stackrel{\circ}{K}) \cap[p, b]$ is closed and non-empty because $X$ is connected. Since $\bigcap_{b \notin K} S_{b}=\emptyset$, we obtain a contradiction with compactness of $K$.

If $p$ is not an extremum, observe that both sets $(\leftarrow, p]$ and $[p, \rightarrow)$ are connected and have $p$ as an accumulation point (with the topology inherited from $X$ ). Therefore, we are led back to the previous situation.

In order to prove that a connected pseudocompact space with a continuous weak selection is orderable, we need the following generalization of $[41,5.5]$.

Proposition 1.19. Every pseudocompact weakly orderable space $X$ is sequentially compact.

Proof. Fix a compatible linear order on $X$. Since every sequence in $X$ has a monotone subsequence, it suffices to show that every strictly increasing sequence $\left\{x_{n}: n \in \omega\right\} \subseteq X$ converges. Choose mutually disjoint open intervals $I_{n}$ in $X$ containing $x_{n}$ and, for each $n$, let $W_{n}=\bigcup_{k \geq n} I_{k}$. By the pseudocompactness of $X$, there exists $p \in \bigcap_{n \in \omega} \bar{W}_{n}$, so that every open interval containing $p$ meets all $W_{n}$ 's. If the sequence $\left\{x_{n}: n \in \omega\right\}$ is increasing, the point $p$ must be its supremum and $\bigcap_{n \in \omega} \bar{W}_{n}=\{p\}$. Assume to the contrary that the sequence $\left\{x_{n}: n \in \omega\right\}$ does not converge. Then there is a closed neighborhood $A$ of $p$ such that $A$ misses infinitely many elements of $\left\{x_{n}: n \in \omega\right\}$. Put $J=\left\{n \in \omega: x_{n} \notin A\right\}$. For every $n \in J$, let $V_{n}=\bigcup\left\{I_{k} \backslash A: k \geq n, k \in J\right\}$. Then $\bigcap_{n \in J} \bar{V}_{n} \subseteq\left(\bigcap_{n \in J} \bar{W}_{n}\right) \backslash\{p\}=\emptyset$, in contradiction with the pseudocompactness of $X$.

Since every connected space with a continuous weak selection is weakly orderable (cf. Corollary 1.23 below) and every connected suborderable space is orderable, Proposition 1.19 and Corollary 1.6 imply the following:

Corollary 1.20. A connected pseudocompact space with a continuous weak selection is orderable.

We now consider separately continuous weak selections, i.e., selections $\sigma$ on $[X]^{2}$ which are separately continuous when considered as maps $\sigma$ : $X \times X \rightarrow X$. This notion is too weak in general. 
EXAMPLE 1.21. Let $\alpha$ be an infinite initial ordinal and let $Y=(\alpha+1, \tau)$ be a topological space on $\alpha+1$ such that all ordinals $\beta<\alpha$ are isolated in $\tau$ and $\tau$-neighborhoods of $\{\alpha\}$ coincide with the ordinal topology, i.e. intervals $\{(\beta, \alpha]: \beta<\alpha\}$ form a local base at $\{\alpha\}$. Put $X=Y \times\{0,1\}$; so $X$ is compact iff $\alpha=\omega_{0}$. Define $\sigma: X \times X \rightarrow X$ as follows: Take two distinct $b=(\beta, i) \in X, c=(\gamma, j) \in X$. Assume $i \leq j$. Let

$$
\sigma(\{b, c\})= \begin{cases}c & \text { if }(i<j \& \beta=\gamma \neq \alpha) \text { or }(i=j \& \beta>\gamma), \\ b & \text { otherwise. }\end{cases}
$$

Then $\sigma$ is separately continuous but it is not continuous.

Proof. Take a map $s: X \times X \rightarrow X$ defined by

$$
s(x, y)= \begin{cases}\sigma(\{x, y\}) & \text { if } x \neq y \\ x & \text { if } x=y\end{cases}
$$

Firstly, the map $s$ is not continuous as $s((\alpha, 0),(\alpha, 1))=(\alpha, 0)$ but $s((y, 0),(y, 1))=(y, 1)$ for $y \in Y \backslash\{\alpha\}$.

The map $s$ is separately continuous: Put $t_{b}(x)=s(b, x)$ for $b \in X, x \in X$. As the map $s$ is symmetric, it is enough to show that $t_{b}$ is continuous on $X$ for any $b \in X$.

Choose $b_{0} \in X, b_{0}=\left(\beta_{0}, i_{0}\right)$. Take $x \in X$ and an open neighborhood $U$ of $z=s\left(b_{0}, x\right)$. Put $x=(\xi, j)$. We should find a neighborhood $V$ of $x$ such that $s\left(b_{0}, y\right) \in U$ for each $y \in V$.

If $b_{0}=x$ then take $V=U$.

If $x$ is isolated in $X$ then take $V=\{x\}$.

So assume $b_{0} \neq x$ and $x$ is not isolated in $X$, in particular $\xi=\alpha$. Then take

$$
V= \begin{cases}\left(\beta_{0}, \alpha\right] \times\{i\} & \text { if } i=j \text { and } z=b_{0} \\ \left((\alpha+1) \backslash\left\{\beta_{0}\right\}\right) \times\{j\} & \text { otherwise. }\end{cases}
$$

For connected spaces, this cannot happen as the next result shows.

Proposition 1.22. Every separately continuous weak selection on a connected space $X$ is continuous.

Proof. Take a representation of a weak selection as a map $\sigma: X \times X \rightarrow X$. For every $x \in X$, we set

$$
B_{x}=\{y \in X: \sigma(x, y)=y\} \quad \text { and } \quad A_{x}=\{y \in X: \sigma(x, y)=x\} .
$$

As $\sigma$ is separately continuous, both $A_{x}$ and $B_{x}$ are closed and $A_{x} \cap B_{x}=\{x\}$ (thus both $A_{x} \backslash\{x\}$ and $B_{x} \backslash\{x\}$ are open). Define a relation $\varrho$ on $X$ by

$$
x \varrho y \Leftrightarrow y \in A_{x} .
$$

To prove that $\varrho$ is a linear order, it suffices to show that $\varrho$ is transitive. Assume that $a, b, c$ are pairwise distinct and $a \varrho b, b \varrho c$. If $c \varrho a$, then $b \in A_{a}$, $c \in A_{b}$ and $a \in A_{c}$. Hence, $a \in B_{b}, b \in B_{c}$. Therefore, for $Z=B_{b} \cap A_{c}$, we have $Z=\left(B_{b} \backslash\{b\}\right) \cap\left(A_{c} \backslash\{c\}\right)$. Since $b \notin Z$ and $a \in Z$, we conclude that $Z$ is 
a proper non-empty clopen subset of the connected space $X$, a contradiction. So, $\varrho$ is transitive. Since $A_{x}$ and $B_{x}$ are closed for every $x \in X$, the identity $\operatorname{map} X \rightarrow(X, \varrho)$ is continuous. As $\sigma(\{x, y\})=\min _{\varrho}\{x, y\}$, the map $\sigma$ is continuous (see [28]).

Proposition 1.22 enables us to derive the following generalization of [28, Lemma 7.2] concerning the equivalence of weak orderability and the existence of a continuous weak selection in connected spaces.

COROLlARY 1.23. If a connected space admits a separately continuous weak selection, then it is weakly orderable.

We now give a generalization of Proposition 1.22 (see [28, Lemma 7.4]).

Proposition 1.24. Suppose that every point of a Hausdorff space $X$ has a connected neighborhood. Then every separately continuous weak selection $\sigma$ on $X$ is continuous.

Proof. The space $X$ is the union of disjoint clopen connected components. Let $C_{x}$ be the component of $x$. If $y \in C_{x}$, then the continuity of $\sigma$ at $\{x, y\}$ follows from Proposition 1.22. If the points $x$ and $y$ belong to distinct components then it suffices to observe that the sets $\left\{x^{\prime} \in C_{x}\right.$ : $\left.\sigma\left(x^{\prime}, y\right)=y\right\},\left\{x^{\prime} \in C_{x}: \sigma\left(x^{\prime}, y\right)=x^{\prime}\right\},\left\{y^{\prime} \in C_{y}: \sigma\left(x, y^{\prime}\right)=y^{\prime}\right\}$ and $\left\{y^{\prime} \in C_{y}: \sigma\left(x, y^{\prime}\right)=x\right\}$ are clopen and, hence, each of them is either empty or coincides with $C_{x}$ or $C_{y}$. Again, this implies the continuity of $\sigma$ at $\{x, y\}$.

Using Corollary 1.23, it is easy to give a rectified extension of the first assertion in [31, Remark 16]. (A subspace of $\mathbb{R}$ obtained as the union of a closed interval with a disjoint open interval shows that suborderability cannot be strengthened to orderability in Corollary 1.25.)

Corollary 1.25. If a locally connected space $X$ has a separately continuous weak selection, then $X$ is suborderable.

Proof. By Corollary 1.23, every component in $X$ is weakly orderable. A connected locally connected weakly orderable space is orderable $[10,8.2]$. The conclusion follows as $X$ is a topological sum of orderable spaces.

1.3. Topological groups. In (locally) pseudocompact topological groups, the existence of a continuous weak selection is very restrictive as the following theorem shows. We recall that a topological group $G$ is said to be topologically orderable if its topology is generated by a linear order (no relation between the group operations and the order is assumed).

THEOREM 1.26. Let $G$ be a locally pseudocompact topological group with a continuous weak selection. Then $G$ is locally compact, metrizable, and topologically orderable. In addition, if $G$ is non-discrete, then it contains an open neighborhood of the identity which is homeomorphic either to the Cantor set or to the reals $\mathbb{R}$. 
Proof. If $G$ is discrete, there is nothing to prove. Hence, we suppose that all neighborhoods of the identity in $G$ are infinite. By our assumption, there exists a pseudocompact neighborhood $F$ of the identity $e$ in $G$. Then the closure $\bar{F}$ of $F$ is also pseudocompact. Denote by $U$ the interior of $\bar{F}$. Then $e \in U \subseteq \bar{F}$. Therefore, the regular closed set $P=\bar{U}$ in the pseudocompact space $\bar{F}$ is also pseudocompact. As the direct product of any number of pseudocompact regular closed subspaces of a topological group remains pseudocompact [39], the space $P^{2}$ is pseudocompact as well. Clearly, $P$ admits a continuous weak selection (being a subspace of $G$ ), so the Stone-Čech compactification $\beta P$ of $P$ is orderable by Theorem 1.16.

Denote by $\widetilde{G}$ the completion of $G$ and let $Q$ be the closure of $P$ in $\widetilde{G}$. Then $P$ is $C$-embedded in $Q$, i.e., $\beta P \cong Q$ (see [8, Theorem 4.2]). We conclude that $Q$ is orderable and, hence, both $Q$ and $P$ have a decreasing well-ordered base at the identity $e$ (cf. [32, Lemma 3]). Since $P$ is a neighborhood of $e$ in $G$, the group $G$ also has a decreasing well-ordered base at $e$. We claim that the cofinality of this base is countable, i.e., the group $G$ is first countable and, hence, metrizable by the Birkhoff-Kakutani theorem. Indeed, otherwise the intersection of each countable family of open sets in $G$ is open, i.e., $G$ is a $P$-space. However, every pseudocompact subspace of a $P$-space is finite, so that $P$ is a finite neighborhood of $e$ in $G$. Therefore, $G$ is discrete, a contradiction.

The pseudocompact subset $P$ of the metrizable group $G$ is compact, whence it follows that $G$ is locally compact. Denote by $H$ the subgroup of $G$ generated by $P$. It is clear that $H$ is open in $G$ (hence, closed). In addition, both $P$ and $H$ are second countable. Indeed, $H$ is $\sigma$-compact as a group generated by the compact set $P$. Therefore, $H$ can be covered by countably many translations of its open second countable subset $U$, whence it immediately follows that $w(H) \leq \omega$.

Suppose first that the connected component $c(G)$ is trivial. Since $G$ is locally compact, it must be zero-dimensional. Then $P$ is a zero-dimensional second countable compact space without isolated points, so that $P$ is homeomorphic to the Cantor set. Since $P$ is a neighborhood of the identity $e$ in $G, e$ has an open neighborhood homeomorphic to the Cantor set. In addition, as $H$ is second countable, there exists a countable disjoint cover of $H$ by clopen sets homeomorphic to the Cantor set, which implies orderability of $H$. It is clear that $G$ is a topological sum of left cosets of its open subgroup $H$, so $G$ is also topologically orderable.

Finally, suppose that $K=c(G) \neq\{e\}$. Then $K \subseteq H$ since $H$ is clopen in $G$. Clearly, $K$ admits a continuous weak selection, being a subspace of $G$. From Theorem 1.17 it follows that the connected space $K$ is weakly orderable. The subgroup $K$ is closed in $G$, hence locally compact. Therefore, $K$ 
is orderable by Proposition 1.18. By [43, Theorem 2.4], $K$ is topologically isomorphic to the additive group $\mathbb{R}$ with the usual interval topology.

Denote by $<$ a linear order on $P$ that generates the original topology of $P$. We claim that there exist points $a, b \in P$ such that $(a, b)$ is a nonempty open subset of $K$. Suppose to the contrary that $(a, b) \backslash K \neq \emptyset$ for every $a, b \in P$ with $(a, b) \neq \emptyset$. Since $K$ is a non-trivial connected subgroup of $G$, the identity $e$ is not isolated in $K$. Clearly, $e$ is in the closure of one of the sets $K \cap(e, \rightarrow)$ or $K \cap(\leftarrow, e)$. Without loss of generality we can assume that $e$ is an accumulation point of $K \cap(e, \rightarrow)$. Choose points $c \in(e, \rightarrow) \backslash K$, $d \in K \cap(e, c)$ and $f \in(e, d) \backslash K$. Then $A=(f, c) \cap K=[f, c] \cap K$ is a clopen subset of $K$ and $d \in A \neq \emptyset$. This contradicts connectedness of $K$, thus proving our claim. Since $U \cap(a, b)$ is a non-empty open subset of $G, K$ must be open in $G$.

We conclude, therefore, that $G$ is a topological sum of clopen subsets homeomorphic to $\mathbb{R}$, so the group $G$ is topologically orderable.

Corollary 1.27. Let $G$ be a pseudocompact topological group with a continuous weak selection. Then $G$ is a topologically orderable compact metrizable totally disconnected group.

Proof. From Theorem 1.26 it follows that $G$ is locally compact, metrizable and topologically orderable. However, every pseudocompact group is precompact [7] and a locally compact precompact group is clearly compact. Total disconnectedness of $G$ follows from [43, Theorem 2.4] which states that a topologically orderable topological group which is not totally disconnected contains an open normal subgroup $H$ homeomorphic to the additive group $\mathbb{R}$ of the reals endowed with the usual topology. The existence of such a subgroup of $G$ is impossible in view of the pseudocompactness of $G$.

Totally disconnected compact metrizable spaces without isolated points are known to be homeomorphic to the Cantor set. Therefore, we have the following:

COROLlary 1.28. Let $X$ be a pseudocompact topological group with a continuous weak selection. Then $X$ is either finite or homeomorphic to the Cantor set.

We finish this section with an example of a weakly orderable but not suborderable topological group. Our group is additionally $\sigma$-compact, zerodimensional and Abelian.

ExAmPlE 1.29. The free Abelian topological group $A(C)$ over the Cantor set $C$ is weakly orderable but not suborderable. 
Proof. Let $d$ be the usual metric on $C$. Then $d$ admits an extension to a continuous invariant metric $\widehat{d}$ over $A(C)$ (cf. [17]). Note that the group $A(C)$ is not metrizable and its topology is strictly finer than the metric topology induced by $\widehat{d}$. Denote by $G$ the metric group $(A(C), \widehat{d})$. It is easy to see that $G$ is second countable and zero-dimensional. Indeed, both $C$ and $A(C)$ are separable. Clearly, the identity map $A(C) \rightarrow G$ is continuous, hence $G$ is also separable. Therefore, $G$ is second countable. Zero-dimensionality of $G$ follows from $\left[1\right.$, Fact $\left.\left(\alpha_{11}\right)\right]$ and the countable sum theorem for the covering dimension in normal spaces. So, $G$ is orderable since every second countable zero-dimensional metric space is orderable ([25], [18] or [22]; the reasoning is quite simplified as dimensions dim, ind and Ind coincide for separable metric spaces, see e.g. [12, Theorem 7.3.3]). Since the identity map $A(C) \rightarrow G$ is continuous, the group $A(C)$ is weakly orderable.

Finally, we claim that $A(C)$ is not suborderable. As noted above, $A(C)$ is separable. If $A(C)$ were suborderable, it would be first countable. But a first countable topological group is metrizable, while metrizability of the free Abelian group $A(X)$ on a space $X$ is equivalent to discreteness of $X$ (cf. [17]).

A simple analysis of the above proof shows that one can take any nondiscrete metrizable space $X$ with $\operatorname{dim} X=0$ instead of the Cantor set $C$ in Example 1.29.

2. Embeddings into ordinals. Scattered spaces may be characterized by the following folklore proposition (see e.g. [36, 8.5.10, Ex. A]):

Proposition 2.1. A Hausdorff space $X$ is scattered if and only if it admits a well-ordering in which initial segments are open. For every such well-ordering, $F \rightarrow \min F$ is a zero-selection for $X$, and every zero-selection gives such a well-ordering.

Proof. Clearly, $X$ is scattered if and only if it has a zero-selection. Let $\varphi$ be such a selection. The required well-ordering on $X$ will be defined by transfinite recursion. Let $x_{0}=\varphi(X)$; then $x_{0}$ is an isolated point. Suppose that we have defined $S_{\alpha}=\left\{x_{\beta}: \beta<\alpha\right\}$ in such a way that the initial segments of $S_{\alpha}$ are open in $X$. The set $S_{\alpha}$ is open because it is the union of its initial segments. If $S_{\alpha} \neq X$, let $Y_{\alpha}=X \backslash S_{\alpha}$ and put $x_{\alpha}=\varphi\left(Y_{\alpha}\right)$. Since $x_{\alpha}$ is isolated in $Y_{\alpha}$, the set $X_{\alpha}=S_{\alpha} \cup\left\{x_{\alpha}\right\}=\left\{x_{\beta}: \beta \leq \alpha\right\}=S_{\alpha+1}$ is still open in $X$.

Obviously, the existence of such a well-ordering implies that $\min F$ is an isolated point of $F$.

The following result (see [28, 7.5.1]) implies that any subspace of an ordinal space has a continuous zero-selection. It is known (see Corollary 2.6 be- 
low) that ordinal spaces are the only spaces with a continuous zero-selection within the realm of compact spaces. Michael's sufficient condition for the existence of a continuous selection may be used for continuous zero-selections:

Lemma 2.2. If $X$ is weakly orderable in such a way that every closed subset of $X$ has a minimum, then the selection $F \rightarrow \min F$ is continuous.

The Fell topology on $\mathfrak{F}(X)$ is the topology generated by the sets of the form $V^{+} \cap W_{1}^{-} \cap \ldots \cap W_{n}^{-}$, where $V, W_{1}, \ldots, W_{n}$ are open subsets of $X$, with the additional condition that $X \backslash V$ is compact. The Fell topology is coarser than the Vietoris topology and they coincide if and only if $X$ is compact. Consequently, every Fell-continuous selection is continuous.

We denote by $[X]^{\leq 2}$ the subset of $\mathfrak{F}(X)$ consisting of the sets with at most two elements. While Vietoris and Fell topologies coincide on $[X]^{2}$, they are different on $[X]^{\leq 2}$. If there exists a Fell-continuous selection on $[X] \leq 2$, then $X$ is locally compact and orderable in such a way that every non-empty closed subset has a minimum [3]. Putting together results from [3] and [21], we obtain:

THEOREM 2.3. The following conditions are equivalent:

(i) $X$ is topologically well-ordered (i.e., $X$ is a linearly ordered topological space such that each non-empty closed set has a minimal element);

(ii) there exists a Fell-continuous selection for $[X]^{\leq 2}$;

(iii) there exists a Fell-continuous selection for $\mathfrak{F}(X)$.

REMARK 2.3.1. It is our pleasant duty to point out that, while finishing this paper, we got acquainted with [20]. The above Theorem 2.3 corresponds to Theorem 1.2 and Corollary 1.3 of [20].

Theorem 2.5 below gives an explicit formulation of a similar equivalence within the class of scattered spaces. First, we need a lemma about convergence of nested sequences of closed sets.

Lemma 2.4. Let $X$ be a topological space and suppose that $\left\{F_{\alpha}: \alpha<\alpha_{0}\right\}$ is a decreasing transfinite sequence of closed subsets of $X$ with the non-empty intersection $F=\bigcap_{\alpha<\alpha_{0}} F_{\alpha}$. The net $\left\{F_{\alpha}: \alpha<\alpha_{0}\right\}$ always converges to $F$ in the Fell topology, and also in the Vietoris topology if some $F_{\alpha}$ is compact $\left({ }^{2}\right)$.

Proof. Since the family $\left\{F_{\alpha}: \alpha<\alpha_{0}\right\}$ is decreasing, it suffices to observe that if an open neighborhood $V$ of $F$ has compact complement, then $F_{\alpha} \subseteq V$ for some $\alpha<\alpha_{0}$. The same occurs if $V$ is an arbitrary open neighborhood of $F$ and some $F_{\alpha}$ is compact.

$\left({ }^{2}\right)$ Clearly, the claim may also be stated for any filter base of closed sets with nonempty intersection. 
THEOREM 2.5. For a Hausdorff space $X$, the following are equivalent:

(i) $X$ is homeomorphic to an ordinal space;

(ii) there exists a Fell-continuous zero-selection for $X$.

Proof. (i) $\Rightarrow$ (ii). It suffices to show that the zero-selection $\varphi(F)=\min F$ is Fell-continuous. If $W$ is an open interval containing $\min F$, then the open set $V=W \cup\{x \in X: x>\min F\}$ contains $F$ and has compact complement. Then $W^{-} \cap V^{+}$is a neighborhood of $F$ in the Fell topology and for every $G \in W^{-} \cap V^{+}$, we have $\min G \in W$.

(ii) $\Rightarrow($ i). Denote by $\varphi$ a Fell-continuous zero-selection on $X$. Let $X=$ $\left\{x_{\alpha}: \alpha<\alpha_{0}\right\}$ be the well-ordering corresponding to $\varphi$, as in Proposition 2.1. Consider the sets $S_{\alpha}, Y_{\alpha}$ and $X_{\alpha}$ defined in the proof of Proposition 2.1. It turns out that the closed set $Y_{\alpha}$ coincides with $\left\{x_{\beta}: \beta \geq \alpha\right\}$. Let $\alpha$ be a limit ordinal. Since $\bigcap_{\beta<\alpha} Y_{\beta}=Y_{\alpha}$, the decreasing net $\left\{Y_{\beta}\right\}_{\beta<\alpha}$ converges to $Y_{\alpha}$ in the Fell topology. By the Fell-continuity of $\varphi$, the sequence $\left\{x_{\beta}\right\}_{\beta<\alpha}$ converges to $x_{\alpha}$ in the topology of $X$. Consequently, every neighborhood of $x_{\alpha}$ contains a neighborhood in the order topology and the order topology is finer than the original one. Finally, since the open subspace $X_{\alpha}$ of $X$ is compact in the order topology, the minimality of compact topologies ensures that the order topology of $X_{\alpha}$ coincides with the subspace topology of $X_{\alpha}$. The conclusion now follows because $X$ is the union of open subspaces $X_{\alpha}$.

Corollary 2.6 ([14, Theorem 1]). A compact space $X$ is homeomorphic to an ordinal space iff there exists a continuous zero-selection for $X$.

Clearly, Theorem 2.5 implies Corollary 2.6 since compactness of $X$ ensures the Fell-continuity of the selection. In Theorem 2.7 below we provide another generalization of Corollary 2.6 weakening the condition of compactness of $X$.

A space $X$ is said to be almost compact if for any two disjoint closed sets in $X$, one of them is compact [15]. One can easily construct a space which is almost compact without being regular. Every almost compact space is countably compact and every regular almost compact space is normal and locally compact. A completely regular space is almost compact iff it has a unique compactification [15]. If $\alpha$ is a limit ordinal, then the ordinal space $\alpha$ is almost compact iff $\alpha$ has uncountable cofinality.

In the proof of the next theorem we use background and notation of Proposition 2.1 and Theorem 2.5. In fact, this theorem provides a topological characterization of ordinal spaces $\alpha$ where $\alpha$ is a successor or has an uncountable cofinality.

THEOREM 2.7. A regular almost compact space $X$ is homeomorphic to an ordinal space if and only if there exists a continuous zero-selection for $X$. 
Proof. The necessity follows from Lemma 2.2 (or by arguing as in Theorem 2.5). For the sufficiency, consider the well-ordering $\left\{x_{\alpha}\right\}$ of $X$ given by the zero-selection. If for every limit ordinal $\alpha$ the net $\left\{Y_{\beta}\right\}_{\beta<\alpha}$ Vietorisconverges to $Y_{\alpha}$, then the conclusion follows as in Theorem 2.5. Otherwise, let $\bar{\alpha}$ be the minimal limit ordinal for which the above net does not converge. Then there exists an open set $\Omega$ in $X$ containing $Y_{\alpha}$ such that $Y_{\beta} \cap(X \backslash \Omega) \neq \emptyset$ for every $\beta<\bar{\alpha}$. Consequently, the closed subset $X \backslash \Omega$ of $X$ is unbounded in $S_{\bar{\alpha}}$. Note that by the minimality of $\bar{\alpha}$, the subspace topology of $S_{\bar{\alpha}}$ coincides with its order topology.

Since the closed set $X \backslash \Omega$ is countably compact (being almost compact), the cofinality of $\bar{\alpha}$ is uncountable. Let $p$ be a point of $Y_{\bar{\alpha}}$ and let $C$ be a closed neighborhood of $p$ with $C \subseteq \Omega$. Then $L=C \cap S_{\bar{\alpha}}$ is a closed subset in the subspace topology of $S_{\bar{\alpha}}$ which is disjoint from $X \backslash \Omega$. Since $X \backslash \Omega$ is unbounded, $L$ must be a bounded closed subset of $S_{\bar{\alpha}}$. Therefore, $L$ is compact and $C \backslash L$ is a neighborhood of $p$ contained in $Y_{\bar{\alpha}}$. This means that $Y_{\bar{\alpha}}$ is clopen, so that $X$ is the topological sum of the clopen subspaces $S_{\bar{\alpha}}$ and $Y_{\bar{\alpha}}$. Since $S_{\bar{\alpha}}$ is not compact, the clopen set $Y_{\bar{\alpha}}$ is compact and, hence, it is homeomorphic to an ordinal space (Corollary 2.6). If $\delta+1$ is the order type of $Y_{\bar{\alpha}}$, then $X$ is homeomorphic to $\delta+1+\bar{\alpha}$.

One may wonder whether almost compactness can be replaced by countable compactness in Theorem 2.7. The answer is negative, as the topological sum of two copies of $\omega_{1}$ shows. However, pseudocompact spaces with a continuous zero-selection admit embeddings into ordinals (Theorem 2.9). We start with a lemma in which $\operatorname{Isol}(X)$ denotes the set of isolated points of $X$.

Lemma 2.8. Let $X$ be a pseudocompact scattered space with a continuous zero-selection and let $\left\{x_{\alpha}\right\}_{\alpha<\delta}$ be the well-ordering given by the selection. Then the topology of $X$ is finer than the ordinal topology of $\delta$, and it is obtained from the latter by declaring isolated some points corresponding to limit ordinals of uncountable cofinality; hence $X$ is suborderable. If $X$ is locally compact, then every non-isolated point of $X$ has a neighborhood $V$ such that $V \cap \operatorname{Isol}(X)$ consists of points corresponding to successor ordinals.

Proof. By Proposition 1.11 and Corollary 1.14, $X$ is countably compact. Consider the open sets $X_{\alpha}, S_{\alpha}$ and the closed set $Y_{\alpha}$ introduced in the proof of Proposition 2.1. We prove the claim by induction on $\alpha$. Suppose that for all $\beta<\alpha$, the set $X_{\beta}$ is closed in $X$ and each non-isolated point $x_{\beta}$ has a neighborhood satisfying the conditions of the lemma. For every $\beta<\alpha$, let $V_{\beta}\left(x_{\alpha}\right)=\left\{x_{\gamma}: \beta<\gamma \leq \alpha\right\}$. By inductive hypothesis, the set $X_{\beta}$ is closed for each $\beta<\alpha$, so $V_{\beta}\left(x_{\alpha}\right)=X_{\alpha} \cap\left(X \backslash X_{\beta}\right)$ is an open neighborhood of $x_{\alpha}$. Let us consider the following possible cases: 
- $\alpha=\gamma+1$. Then the set $X_{\alpha}=X_{\gamma} \cup\left\{x_{\alpha}\right\}$ is closed and $\left\{x_{\alpha}\right\}=V_{\gamma}\left(x_{\alpha}\right)$ is open.

- $\alpha$ is limit and the net $\left\{Y_{\beta}\right\}_{\beta<\alpha}$ converges to $Y_{\alpha}$ in the Vietoris topology. Then every neighborhood $V$ of $x_{\alpha}$ contains some $V_{\beta}\left(x_{\alpha}\right)$. Therefore, the neighborhood system of $x_{\alpha}$ in the topology of $X$ coincides with the neighborhood system of $x_{\alpha}$ in the order topology. Let $W$ be a neighborhood of $x_{\sigma}$ disjoint from some $V_{\beta}\left(x_{\alpha}\right)$, where $\sigma>\alpha$. Then $W \cap\left(X \backslash X_{\beta}\right)$ is a neighborhood of $x_{\sigma}$ disjoint from $X_{\alpha}$ and $X_{\alpha}$ is closed.

- $\alpha$ is limit and the net $\left\{Y_{\beta}\right\}_{\beta<\alpha}$ does not Vietoris-converge to $Y_{\alpha}$. Then there exists an open subset $\Omega$ containing $Y_{\alpha}$ such that $Y_{\beta} \cap(X \backslash \Omega) \neq \emptyset$ for every $\beta<\alpha$. Thus, $X \backslash \Omega \subseteq S_{\alpha}$ is a closed subset of $X$ which is unbounded in $S_{\alpha}$. If $\alpha$ has countable cofinality, take a sequence $\beta_{n} \nearrow \alpha$. Then $Y_{\beta_{n}} \cap$ $(X \backslash \Omega) \neq \emptyset$ for each $n \in \omega$, and $\left(\bigcap_{n \in \omega} Y_{\beta_{n}}\right) \cap(X \backslash \Omega)=Y_{\alpha} \cap(X \backslash \Omega)=\emptyset$, in contradiction with countable compactness of $X$. Consequently, the cofinality of $\alpha$ is uncountable.

Let $L$ be a closed subset of $S_{\alpha}$ such that $L \cap\left(X \backslash X_{\beta}\right) \neq \emptyset$ for every $\beta<\alpha$. We want to prove that $L \cap(X \backslash \Omega) \neq \emptyset$. Choose points $y_{1}<z_{1}<y_{2}<z_{2}<\ldots$ such that $y_{i} \in L$ and $z_{i} \in X \backslash \Omega$ for each $i \geq 1$. If $w$ is the common supremum of these sequences in the order of $\delta$, we have $w \in S_{\alpha}$. Since $w$ has countable cofinality, the inductive hypothesis implies that $w \in L \cap(X \backslash \Omega)$.

Now, for $\sigma \geq \alpha$, let $V$ be a closed neighborhood of $x_{\sigma}$ such that $V \subseteq$ $X_{\sigma} \cap \Omega$. Then $L=V \cap S_{\alpha}$ is contained in some $X_{\beta}$ with $\beta<\alpha$. Thus, $V \backslash X_{\beta}$ is a neighborhood of $x_{\sigma}$ contained in $Y_{\alpha} \cap X_{\sigma}$. Consequently, $x_{\alpha}$ is isolated (take $\sigma=\alpha$ ) and $X_{\alpha}$ is closed (take $\sigma>\alpha$ and $x_{\alpha} \notin V$ ).

To prove the last assertion of the lemma, suppose that $X$ is locally compact and let $x_{\alpha}$ be a non-isolated point of $X$. Since the sets $V_{\beta}\left(x_{\alpha}\right)=$ $X_{\alpha} \cap Y_{\beta+1}$ form a base of closed neighborhoods of $x_{\alpha}$, there exists $\beta<\alpha$ such that $V_{\beta}\left(x_{\alpha}\right)$ is compact. Suppose that there exists a limit ordinal $\sigma$ with $\beta<\sigma<\alpha$ such that $x_{\sigma}$ is an isolated point of $X$. Then $C_{\gamma}=V_{\gamma}\left(x_{\sigma}\right) \backslash\left\{x_{\sigma}\right\}$ is a closed non-empty subset of $V_{\beta}\left(x_{\alpha}\right)$ for every $\gamma$ with $\beta<\gamma<\sigma$. Since $\bigcap_{\gamma<\sigma} C_{\gamma}=\emptyset$, we get a contradiction.

THEOREM 2.9. Let $X$ be a pseudocompact space. If there exists a continuous zero-selection for $X$, then $X$ is homeomorphic to a sequentially compact subspace of an ordinal space. This subspace is open if $X$ is locally compact.

Proof. Let $\left\{x_{\alpha}\right\}_{\alpha<\delta}$ be as in Lemma 2.8. Represent every ordinal in the form $\alpha+n$, where $\alpha$ is a limit ordinal and $n<\omega$. Consider the map $\iota: X \rightarrow \delta+1$ defined by

$$
\iota\left(x_{\alpha+n}\right)= \begin{cases}\alpha+n & \text { if } x_{\alpha} \text { is an accumulation point of } X, \\ \alpha+n+1 & \text { if } x_{\alpha} \text { is an isolated point of } X .\end{cases}
$$


Using Lemma 2.8, it is straightforward to verify that the map $\iota$ is the required embedding.

We finish this section with a result about zero-selections for subspaces. Actually, we do not know whether normality can be dropped in the next proposition. This is also connected with Conjecture 5.4: if the conjecture were true, the assumption of normality in Proposition 2.10 would not be necessary.

Proposition 2.10. Let $Y$ be a subspace of a normal space $X$. If $\varphi$ is a continuous zero-selection for $X$, then the map $\psi$ defined by $\psi(F)=\varphi(\bar{F})$ for each relatively closed set $F \subseteq Y$ is a continuous zero-selection for $Y$.

Proof. Let $F$ be a relatively closed subset of $Y$ and put $q=\varphi(\bar{F})$. Since $q$ is an isolated point of $\bar{F}$, we have $q \in F$. To check the continuity of $\psi$, let $W$ be a neighborhood of $q$ in $X$. Let $\mathcal{Z}=\Omega^{+} \cap V_{1}^{-} \cap \ldots \cap V_{n}^{-}$be a neighborhood of $\bar{F}$ in $\mathfrak{F}(X)$ such that if $B \in \mathcal{Z}$ then $\varphi(B) \in W$. Since $\bar{F} \subseteq \Omega$, take in $X$ an open subset $U$ such that $\bar{F} \subseteq U \subseteq \bar{U} \subseteq \Omega$. Then $\mathcal{Z}_{Y}=(U \cap Y)^{+} \cap\left(V_{1} \cap Y\right)^{-} \cap \ldots \cap\left(V_{n} \cap Y\right)^{-}$is a neighborhood of $F$ in $\mathfrak{F}(Y)$. If $G$ belongs to $\mathcal{Z}_{Y}$, it is straightforward to prove that $\bar{G} \in \mathcal{Z}$ and, thus, $\psi(G)=\varphi(\bar{G}) \in W \cap Y$.

3. Covering properties may be helpful. It is worth noting that zero-selections are not always necessary when describing (open) subsets of ordinals: e.g., every scattered first countable compact space is homeomorphic to a countable successor ordinal (Mazurkiewicz-Sierpiński, [36]). By this theorem, every compact countable space, being scattered and metrizable, is homeomorphic to a countable ordinal. Consequently, every locally compact locally countable space, LCC-space for short, is scattered and zerodimensional. Another related result is due to Telgársky who proved that a first countable scattered paracompact space is completely metrizable and embeddable into an ordinal space [37]. Below we present several theorems which generalize these results and extend them to spaces having nice covering properties.

Let us recall that a scattered space $X$ can be represented in the form $\bigcup_{\alpha<\lambda} L^{\alpha}$ for some ordinal $\lambda$, where, for $\alpha<\lambda, L^{\alpha}$ is the non-empty set of all isolated points of $X \backslash \bigcup_{\beta<\alpha} L^{\beta}$ (e.g., $L^{0}$ is the set of isolated points of $X$ ). The height $h(X)$ of $X$ is $\lambda$, the $\alpha$ th level is $L^{\alpha}$ and the rank of $p \in X$ is the $\alpha<\lambda$ for which $p \in L^{\alpha}$. For every $p \in L^{\alpha}$, there exists a neighborhood $V_{p}$ of $p$ with $V_{p} \subseteq \bigcup_{\beta \leq \alpha} L^{\beta}$ and $V_{p} \cap L^{\alpha}=\{p\}$; such a neighborhood is said to be a topped neighborhood of $p$. Observe that $L^{\alpha}$ is a closed and discrete subset of $\bigcup_{\beta \leq \alpha} L^{\beta}$. Every locally compact scattered space is zero-dimensional [36, Theorem 8.5.4]. 
A space is said to be ultraparacompact if every open covering has a disjoint open refinement. Since every paracompact scattered space is ultraparacompact [37, 35], we obtain the following result:

TheOREm 3.1. Let $X$ be a locally compact paracompact space. If $X$ has a continuous zero-selection, then it is homeomorphic to a topological sum of successor ordinals. Consequently, it is homeomorphic to an open subspace of an ordinal space. If $X$ is Lindelöf, then it is homeomorphic to an ordinal space of countable cofinality.

Proof. Take a cover $\mathcal{U}$ of $X$ consisting of open sets with compact closure. By [37], there exists a disjoint open refinement $\mathcal{V}$ of this covering. Every $V \in \mathcal{V}$ is clopen and compact as a subset of some $\bar{U}, U \in \mathcal{U}$. Since every $V \in \mathcal{V}$ has a continuous zero-selection, every element of this partition is homeomorphic to a compact ordinal space by Corollary 2.6.

If $X$ is Lindelöf, it suffices to observe that a countable topological sum of compact ordinal spaces is homeomorphic to an ordinal space of countable cofinality.

REMARK 3.1.1. Let $S$ be an ordinal equipped with a locally compact topology $\tau$ which is finer than the order topology. Then there exists a continuous zero-selection for $(S, \tau)$ (e.g., see Lemma 2.2). If $S$ is countable, then $S$ is Lindelöf; consequently, $(S, \tau)$ is homeomorphic to a countable ordinal space. Under the assumption of $\diamond$, in Theorem 4.6 below, we construct a topology $\tau$ on $\omega_{1}$ for which the space $\left(\omega_{1}, \tau\right)$ is monotonically normal, locally compact, locally countable and not suborderable.

We give a positive result for locally compact scattered spaces of countable height which is interesting in comparison with a counterexample promised in Remark 3.1.1.

Recall that a space $X$ is called strongly collectionwise Hausdorff if for every closed discrete subset $L$ of $X$, there exists a discrete family $\left\{U_{x}\right\}_{x \in L}$ of open subsets of $X$ such that $x \in U_{x}$ for every $x \in L$. A collectionwise normal space is strongly collectionwise Hausdorff. A space is said to be meta-Lindelöf if every open cover has an open point-countable refinement.

Lemma 3.2. Let $X$ be a regular scattered space.

(1) If $X$ is hereditarily strongly collectionwise Hausdorff and $h(X)<\omega_{1}$, then $X$ is hereditarily meta-Lindelöf.

(2) If $X$ is strongly collectionwise Hausdorff and $h(X)$ is finite, then $X$ is ultraparacompact.

Proof. (1) It suffices to prove that $X$ is meta-Lindelöf. We proceed by transfinite induction on the height of $X$. If $h(X)=1$, then $X$ is discrete. Let $h(X)=\alpha$ and suppose that the conclusion holds for every space $Y$ satisfying 
the hypotheses with $h(Y)<\alpha$. We will prove that every open covering $\mathcal{A}$ of $X$ has a point-countable open refinement.

If $\alpha=\gamma+1$, then the maximal level $L^{\gamma}$ is closed and discrete. For every $p \in L^{\gamma}$, choose an open neighborhood $U_{p}$ of $p$ in such a way that the family $\left\{U_{p}\right\}_{p \in L^{\gamma}}$ is discrete and each $U_{p}$ is contained in some member of $\mathcal{A}$. Then choose a closed neighborhood $V_{p}$ of $p$ such that $V_{p} \subseteq U_{p}$ for every $p \in L^{\gamma}$. Consider the open set $M=X \backslash \bigcup_{p \in L^{\gamma}} V_{p}$. Its height is at most $\gamma$, so by the inductive hypothesis, $M$ is meta-Lindelöf. Therefore, there exists a point-countable family $\mathcal{W}$ of open subsets of $M$ such that $\bigcup \mathcal{W}=M$ and $\mathcal{W}$ refines $\mathcal{A} \mid M$. Thus, the cover $\mathcal{W} \cup\left\{U_{p}: p \in L^{\gamma}\right\}$ is an open point-countable refinement of $\mathcal{A}$.

If $\alpha$ is limit, then $X=\bigcup_{\beta<\alpha} L^{\beta}$. By the inductive hypothesis, the open subspace $\bigcup_{\gamma \leq \beta} L^{\gamma}$ is meta-Lindelöf for every $\beta$. Consequently, since $\alpha$ is countable, the space $X$ is meta-Lindelöf because it coincides with a countable union of open meta-Lindelöf subspaces.

(2) It suffices to prove that $X$ is paracompact [37]. We proceed by induction on the height of $X$. If $h(X)=1$, then $X$ is discrete. Let $h(X)=n$ and suppose that the statement holds for every space $Y$ satisfying the hypotheses with $h(Y)<n$. The maximal level $L^{n}$ is closed and discrete in $X$. We will prove that every open cover $\mathcal{A}$ of $X$ has a locally finite refinement ([12, Theorem 5.1.11]; recall that this refinement may consist of arbitrary sets). For every $p \in L^{n}$, choose an open neighborhood $U_{p}$ of $p$ in such a way that the family $\left\{U_{p}\right\}_{p \in L^{n}}$ is discrete and each $U_{p}$ is contained in some member of $\mathcal{A}$. Consider the closed set $M^{n}=X \backslash \bigcup_{p \in L^{n}} U_{p}$. Its height is smaller than $n$ and it is strongly collectionwise Hausdorff. By the inductive hypothesis, $M^{n}$ is paracompact. Therefore, there exists a locally finite (in $M^{n}$ ) cover $\mathcal{W}$ of $M^{n}$ which refines $\mathcal{A} \mid M^{n}$. It is not restrictive to assume that $\mathcal{W}$ consists of subsets of $M^{n}$. As $M^{n}$ is closed, it follows that $\mathcal{W}$ is locally finite in $X$. Thus, the cover $\mathcal{W} \cup\left\{U_{p}: p \in L^{n}\right\}$ is the required locally finite refinement of $\mathcal{A}$.

REMARK 3.2.1. (1) The first statement of Lemma 3.2 is not true without the assumption on the height of $X$ as the ordinal space $\omega_{1}$ shows. In addition, by Theorem 3.1, the example provided in Theorem 4.7 below shows that the second statement of Lemma 3.2 does not hold for spaces of countable height.

(2) Lemma 3.2 is sufficiently strong for our purpose. For example, one can modify (1) in Lemma 3.2 adding the assumption that $X$ is locally c.c.c.: then $X$ will be paracompact.

Proposition 3.3. If $X$ is a meta-Lindelof $L C C$-space, then $X$ is metrizable and homeomorphic to an open subspace of an ordinal.

Proof. Let $\mathcal{A}$ be a point-countable open cover of $X$ consisting of countable sets with compact closure. Consider the sets $\mathrm{St}^{\omega}(A)=\bigcup_{n \in \omega} \operatorname{St}^{n}(A)$, 
with $A \in \mathcal{A}$, which form a disjoint clopen cover of $X$. Every $\operatorname{St}^{\omega}(A)$ is countable and locally compact, hence Lindelöf and scattered. Therefore, it is ultraparacompact [37], so it may be split into a sum of compact countable spaces, which are homeomorphic to countable ordinals. Since compact countable spaces are metrizable, so is $X$.

Lemma 3.2 and Proposition 3.3 imply:

THEOREM 3.4. Let $X$ be an LCC-space with the following properties:

(1) $h(X)<\omega_{1}$

(2) $X$ is hereditarily strongly collectionwise Hausdorff.

Then $X$ is metrizable and homeomorphic to an open subspace of an ordinal space.

Every suborderable space is monotonically normal and, hence, hereditarily collectionwise normal (see [19]). One may ask if condition (1) on the height of $X$ can be omitted in Theorem 3.4. Assuming the Diamond Principle $\diamond$, we will construct a monotonically normal LCC-space $X$ which has a continuous zero-selection, but it is not suborderable (Theorem 4.6). This example shows that the assumptions of Proposition 3.3 and Theorem 3.1 cannot be easily weakened. The second claim in Lemma 3.2 implies the following fact close to Theorem 3.4.

TheOrem 3.5. Let $X$ be an LCC-space with $h(X)<\omega$. If $X$ is strongly collectionwise Hausdorff, then $X$ is metrizable and homeomorphic to an open subspace of an ordinal space.

Theorem 4.7 in the next section shows that the above result is no more valid for spaces $X$ with $h(X)=\omega$.

4. Counterexamples. We need some preliminaries. A subset $C$ of $\omega_{1}$ is said to be a $c u b$ in $\omega_{1}$ if $C$ is closed in the order topology and unbounded in $\omega_{1}$. Clearly, the set $\operatorname{Lim}\left(\omega_{1}\right)$ of all limit ordinals less than $\omega_{1}$ is a cub. The intersection of countably many cubs is again a cub (see [11] or [23]). We say that $B \subseteq \omega_{1}$ is stationary if $B \cap C \neq \emptyset$ for every cub $C$. The intersection of a cub with a stationary set is again a stationary set. We also put $\operatorname{Isol}\left(\omega_{1}\right)=\omega_{1} \backslash \operatorname{Lim}\left(\omega_{1}\right)$.

Let $B \subseteq \omega_{1}$. A function $\vartheta: B \rightarrow \omega_{1}$ is called regressive if $\vartheta(\alpha)<\alpha$ for every $\alpha \in B$.

Lemma 4.1 (Fodor). Let $\vartheta: B \rightarrow \omega_{1}$ be a regressive function, where $B$ is a stationary subset of $\omega_{1}$. Then there is a stationary set $B^{\prime} \subseteq B$ such that $\vartheta$ is constant on $B^{\prime}$.

Corollary 4.2. For every unbounded subset $B$ of $\omega_{1}$, the set $C=$ $\left\{\alpha<\omega_{1}: \sup (B \cap \alpha)=\alpha\right\}$ is a cub. 
Our constructions depend on a set-theoretic assumption consistent with ZFC and known as the Diamond Principle.

DiAmond PRINCIPLE. There exists a transfinite sequence $\left\{A_{\alpha}: \alpha<\omega_{1}\right\}$ of subsets of $\omega_{1}$ such that $A_{\alpha} \subseteq \alpha$ for each $\alpha<\omega_{1}$ and, for every subset $B$ of $\omega_{1}$, the set $\left\{\alpha<\omega_{1}: B \cap \alpha=A_{\alpha}\right\}$ is stationary. Such a sequence $\left\{A_{\alpha}: \alpha<\omega_{1}\right\}$ will be called $a \diamond$-sequence.

Notice that the set $A=\left\{\alpha<\omega_{1}: \sup A_{\alpha}=\alpha\right\}$ is stationary for every $\diamond$-sequence $\left\{A_{\alpha}\right\}_{\alpha<\omega_{1}}$. We abbreviate the diamond principle to $\diamond$ (see [23] or [11]). Let us introduce notation which will be used in this section.

Notation 4.3. Let $\left\{A_{\alpha}: \alpha<\omega_{1}\right\}$ be a $\diamond$-sequence. Set $A=\left\{\alpha<\omega_{1}\right.$ : $\left.\sup A_{\alpha}=\alpha\right\}$. For an unbounded subset $B$ of $\omega_{1}$, put

$$
A(B)=\left\{\alpha \in A: B \cap \alpha=A_{\alpha}\right\} \quad \text { and } \quad I(B)=\left\{\alpha \in A: B \supset A_{\alpha}\right\} .
$$

Proposition 4.4. If $\left\{A_{\alpha}: \alpha<\omega_{1}\right\}$ is a $\diamond$-sequence and $B \subseteq \omega_{1}$ is unbounded, then both sets $A(B)$ and $I(B)$ are stationary.

We omit the proofs of Corollary 4.2 and Proposition 4.4 as they are well known and pretty standard. We shall use the following characterization of monotonically normal spaces given in [19, Theorem 5.19]:

Lemma 4.5. A space $X$ is monotonically normal iff to each open set $U \subset X$ and $x \in U$, one can assign an open set $U_{x}$ contained in $U$, containing $x$ and satisfying the following condition:

$$
U_{x} \cap V_{y} \neq \emptyset \quad \text { implies } \quad x \in V \text { or } y \in U .
$$

We are finally ready to present the first counterexample.

TheOREm 4.6. Assume $\diamond$. There exists a topology $\tau$ on the set $\omega_{1}$, finer than the order topology, such that $X=\left(\omega_{1}, \tau\right)$ is a monotonically normal $L C C$-space with a continuous zero-selection, but $X$ is not suborderable.

Proof. First, we construct the topology $\tau$ on $\omega_{1}$ and then we prove in several steps that the space $X=\left(\omega_{1}, \tau\right)$ is as required.

Apply the diamond principle to take a $\diamond$-sequence $\left\{A_{\alpha}: \alpha<\omega_{1}\right\}$ and define $D=\left\{\alpha \in \operatorname{Lim}\left(\omega_{1}\right): \sup \left(A_{\alpha} \cap \operatorname{Isol}\left(\omega_{1}\right)\right)=\alpha\right\}$. The set $D$ is stationary because it contains $A\left(\operatorname{Isol}\left(\omega_{1}\right)\right)$ (see Proposition 4.4). If $\alpha \in D$, then choose $\left\{\sigma^{\alpha}(n)\right\}_{n \in \omega} \subseteq A_{\alpha} \cap \operatorname{Isol}\left(\omega_{1}\right)$ such that

(1) $\sigma^{\alpha}(n) \nearrow \alpha$;

(2) if $\sigma^{\alpha}(3 n+1)$ has a successor $s$ in $A_{\alpha}$ such that $s \in \operatorname{Isol}\left(\omega_{1}\right)$, then $\sigma^{\alpha}(3 n+2)=s$ (this requirement is used to prove Fact 3 below).

For $\delta<\alpha$, put

$$
\begin{aligned}
& L_{0}^{\alpha}=\left\{\sigma^{\alpha}(3 n): n \in \omega\right\}, \\
& C_{\delta}^{\alpha}=(\delta, \alpha) \cap \bigcup\left\{\left\{\sigma^{\alpha}(3 n+1), \sigma^{\alpha}(3 n+2)\right\}: n \in \omega\right\} .
\end{aligned}
$$


If $\alpha \notin D$ and $\delta<\alpha$, then put $L_{0}^{\alpha}=C_{\delta}^{\delta}=\emptyset$.

Recursive construction of $\tau$. For each $\alpha \in \omega_{1}$, the family $\mathcal{B}(\alpha)$ will be a local base of topped neighborhoods of $\alpha$ for a topology $\tau$ which is finer than the order topology of $\omega_{1}$. On $\alpha \cup\{\alpha\}$, we consider the topology $\tau_{\alpha}$ generated by $\bigcup_{\beta<\alpha} \mathcal{B}(\beta)$. The topology $\tau$ will have the union of all $\tau_{\alpha}$ 's as a base, and $\tau$ will induce on $\alpha \cup\{\alpha\}$ the topology $\tau_{\alpha}$. In addition, the family $\mathcal{B}(\alpha)$ will satisfy the following condition:

$\left(I_{\alpha}\right) \quad \mathcal{B}(\alpha)=\left\{U_{\gamma}(\alpha): \gamma<\alpha\right\}$, where each $U_{\gamma}(\alpha)$ is a compact open neighborhood of $\alpha$ (in $\tau_{\alpha}$ ) such that $U_{\gamma}(\alpha) \subseteq(\gamma, \alpha]$ and $U_{\gamma}(\alpha) \cap$ $\operatorname{Lim}\left(\omega_{1}\right)=(\gamma, \alpha] \cap \operatorname{Lim}\left(\omega_{1}\right)$ whenever $\alpha \in \operatorname{Lim}\left(\omega_{1}\right)$, and $\mathcal{B}(\alpha)=$ $\{\{\alpha\}\}$ whenever $\alpha \in \operatorname{Isol}\left(\omega_{1}\right)$.

The topology $\tau$ is defined as follows. Suppose that $\tau_{\gamma}$ 's have been defined for all $\gamma<\alpha \in \omega_{1}$ in such a way that $\left(I_{\gamma}\right)$ holds for all $\gamma<\alpha$.

- If $\alpha \in \operatorname{Isol}\left(\omega_{1}\right)$, then $\{\alpha\}$ is an isolated point in $\tau_{\alpha}$ (hence, in $\tau$ ) and $\mathcal{B}(\alpha)=\{\{\alpha\}\}$.

- If $\alpha \in \operatorname{Lim}\left(\omega_{1}\right)$, then it is convenient to distinguish two cases:

(1) $\alpha$ is not isolated in $\operatorname{Lim}\left(\omega_{1}\right)$, i.e. there is a sequence $\left\{\beta_{n}\right\}_{n<\omega}$ such that $\beta_{n} \nearrow \alpha$ and $\beta_{n} \in \operatorname{Lim}\left(\omega_{1}\right)$ for each $n \in \omega$;

(2) $\alpha$ is isolated in $\operatorname{Lim}\left(\omega_{1}\right)$.

To make our notation less clumsy, we put $\beta_{-1}=0$.

Ad (1). We take a sequence $\left\{\beta_{n}\right\}_{n<\omega}$ as in (1). For every $n \in \omega$, choose the neighborhood $W_{n}=U_{\beta_{n-1}}\left(\beta_{n}\right)$ of $\beta_{n}$. By $\left(I_{\beta_{n}}\right), W_{n}$ is compact and open in $\tau_{\beta_{n}}, W_{n} \subset\left(\beta_{n-1}, \beta_{n}\right]$ and $W_{n} \cap \operatorname{Lim}\left(\omega_{1}\right)=\left(\beta_{n-1}, \beta_{n}\right] \cap \operatorname{Lim}\left(\omega_{1}\right)$ for each $n \in \omega$.

Ad (2). If $\alpha>\omega$, put $\beta_{0}=\max \left(\alpha \cap \operatorname{Lim}\left(\omega_{1}\right)\right)$. Then we take $\left\{\beta_{n}\right\}_{n=1}^{\infty}$ as the increasing enumeration of all ordinals in $\left(\beta_{0}, \alpha\right)$. We put $W_{0}=U_{0}\left(\beta_{0}\right)$ and $W_{n}=\left\{\beta_{n}\right\}$ for $n \geq 1$. If $\alpha=\omega$, put $\beta_{n}=n+1$ and $W_{n}=\left\{\beta_{n}\right\}$ for every $n \in \omega$.

Definition of $\mathcal{B}(\alpha)$. Whichever case occurs, choose $\delta<\alpha$. So there exists $n_{\delta} \in \omega$ such that $\delta \in\left[\beta_{n_{\delta}-1}, \beta_{n_{\delta}}\right)$. Put $U_{\delta}=U_{\delta}\left(\beta_{n_{\delta}}\right)$ provided case (1) occurs or $n_{\delta}=0 \& \alpha>\omega$ in case (2). Put $U_{\delta}=\emptyset$ otherwise. Then put $U_{\delta}(\alpha)=$ $\left(\{\alpha\} \cup U_{\delta} \cup\left(\bigcup_{k=n_{\delta}+1}^{\infty} W_{k}\right) \cup C_{\delta}^{\alpha}\right) \backslash L_{0}^{\alpha}$. Finally, set $\mathcal{B}(\alpha)=\left\{U_{\delta}(\alpha): \delta<\alpha\right\}$.

Note that if $C_{0}^{\alpha}$ is non-empty, then it $\tau_{\alpha}$-converges to $\alpha$, and $L_{0}^{\alpha}$ is closed and discrete. It is easy to see that $\mathcal{B}(\alpha)$ satisfies $\left(I_{\alpha}\right)$, and this completes our recursive construction.

Clearly, the family $\bigcup_{\alpha<\omega_{1}} \mathcal{B}(\alpha)$ is a base for the topology $\tau$ on $\omega_{1}$ which is finer than the order topology of $\omega_{1}$, and $\tau$ is zero-dimensional, locally compact and locally countable. In other words, the space $X=\left(\omega_{1}, \tau\right)$ is LCC. 
For the later references, we sum up some properties of $\tau$ :

FACT 1. The restriction of $\tau$ to $\operatorname{Lim}\left(\omega_{1}\right)$ coincides with the order topology of $\operatorname{Lim}\left(\omega_{1}\right)$ and $\operatorname{Isol}\left(\omega_{1}\right)$ is dense in $X$. In addition, $X$ has a continuous zero-selection by Remark 3.1.1.

FACT 2. $(X, \tau)$ is monotonically normal.

Proof. We are going to use Lemma 4.5. For an open set $U \subset X$ and $x \in U$, put $U_{x}=\{x\}$ if $x$ is isolated. If $x$ is not isolated, take an element $U_{x} \in \mathcal{B}(x)$ such that $U_{x} \subset U$. Assume that $U_{x} \cap V_{y} \neq \emptyset$ for open subsets $U, V$ of $X$. We may suppose that $x \neq y$. Let us show that $x \in V$ or $y \in U$. If $x$ or $y$ is isolated, we are done. So assume that neither $x$ nor $y$ is isolated and $x<y$. Then there is $z<x$ such that $z \in U_{x} \cap V_{y}$. By $\left(I_{y}\right)$ and as $V_{y} \subset V$, we obtain $x \in V$. This proves Fact 2 .

FACT 3. Let $B$ and $C$ be uncountable subsets of $\omega_{1}$. Then $\bar{B}^{\tau} \cap \bar{C}^{\tau}$ is uncountable, even stationary.

Proof. There exist sequences $E=\left\{b_{\alpha}: \alpha<\omega_{1}\right\}$ and $F=\left\{c_{\alpha}: \alpha<\omega_{1}\right\}$ such that $b_{\alpha} \in B, c_{\alpha} \in C$ and $b_{\alpha}<c_{\alpha}<b_{\alpha+1}$ for each $\alpha<\omega_{1}$. The sets $E, F$ and $T=E \cup F$ are unbounded in $\omega_{1}$. Observe that if $x \in E$, then the successor of $x$ in the order of $T$ belongs to $F$. It is not restrictive to assume that one of the next three cases occurs:

(1) $B \cup C \subseteq \operatorname{Isol}\left(\omega_{1}\right)$. Let $A(T) \subseteq D$ be the stationary set such that $T \cap \alpha=A_{\alpha}$ and $\sup A_{\alpha}=\alpha$ for every $\alpha \in A(T)$ (see Proposition 4.4). We claim that if $\alpha \in A(T)$, then $\alpha \in \bar{B}^{\tau} \cap \bar{C}^{\tau}$. Since $\alpha \in D$, the sequence $C_{0}^{\alpha}$ is non-empty, is contained in $T$ and $\tau$-converges to $\alpha$. In addition, $\sigma^{\alpha}(3 n+1)$ $\in E$ iff $\sigma^{\alpha}(3 n+2) \in F$, so we have $\alpha \in \bar{B}^{\tau} \cap \bar{C}^{\tau}$.

(2) $B \cup C \subseteq \operatorname{Lim}\left(\omega_{1}\right)$. In this case $\bar{B}^{\tau}$ and $\bar{C}^{\tau}$ are closed in the order topology and the intersection is a cub.

(3) $B \subseteq \operatorname{Isol}\left(\omega_{1}\right)$ and $C \subseteq \operatorname{Lim}\left(\omega_{1}\right)$. As in case (1), the stationary set $A(B)$ is contained in $\bar{B}^{\tau}$. The conclusion now follows since $\bar{C}^{\tau}$ is a cub.

Fact 3 is proved.

We now show that $X$ is not suborderable. By a result of Purisch [34], a scattered suborderable space is orderable, so it suffices to prove the following:

FACT 4. The space $X$ is not orderable.

Proof. Suppose to the contrary that there exists a linear order $\varrho$ on $X$ inducing the topology $\tau$. We divide the proof into several steps.

Claim 1. There is no uncountable subset $M$ of $\operatorname{Isol}\left(\omega_{1}\right)$ such that the restriction of $\varrho$ or $\varrho^{-1}$ to $M$ coincides with the well-order on $M$ inherited from $\omega_{1}$. 
Indeed, assume that $\left.\varrho\right|_{M}$ coincides with the standard order on $M$. Take $\alpha \in A(M)$. Then $\alpha \in D$ and $M \cap \alpha=A_{\alpha}$. The sequence $C_{0}^{\alpha} \tau$-converges to $\alpha$. The definition of the order topology implies that $L_{0}^{\alpha} \varrho$-converges to $\alpha$, a contradiction. The case of $\varrho^{-1}$ is similar.

In what follows, by $\varrho$-intervals we shall mean the sets $[a, b]_{\varrho},(\leftarrow, b]_{\varrho}$ or $[a, \rightarrow)_{\varrho}$, where $a, b \in X$, or the whole $X$.

Claim 2. For each $x \in X$, either $(\leftarrow, x]_{\varrho}$ or $[x, \rightarrow)_{\varrho}$ is countable.

This follows from Fact 3 .

Claim 3. If $\Delta$ is the intersection of countably many @-intervals and $\Delta$ is uncountable, then there is $y \in X$ such that $[y, \rightarrow)_{\omega_{1}} \subseteq \Delta$.

This follows from Claim 2.

Let us return to the proof of Fact 4. We consider two possibilities:

CASE 1 . There is an uncountable $\varrho$-interval $J$ such that the $\varrho$-interval $[a, b]_{\varrho}$ is countable for all $a, b \in \operatorname{int}_{\varrho} J\left(^{3}\right)$.

CASE 2. For each uncountable $\varrho$-interval $J$, there is a $\varrho$-interval $[a, b]_{\varrho}$, with $a, b \in \operatorname{int}_{\varrho} J$, such that $[a, b]_{\varrho}$ is uncountable.

Suppose that Case 1 holds. By recursion, one can define an uncountable subset $M$ of Isol $\left(\omega_{1}\right)$ such that either $\left.\varrho\right|_{M}$ or $\left.\varrho^{-1}\right|_{M}$ coincides with the order on $M$ inherited from $\omega_{1}$, so Claim 1 yields a contradiction. Indeed, one knows from the beginning of the inductive construction whether $\varrho$ or $\varrho^{-1}$ works: take $\gamma_{0} \in \operatorname{Isol}\left(\omega_{1}\right) \cap \operatorname{int}_{\varrho} J$ such that $\left[\gamma_{0}, \rightarrow\right)_{\omega_{1}} \subseteq J$. If the $\varrho$-interval $\left[\gamma_{0}, \rightarrow\right)_{\varrho}$ is uncountable, then $\varrho$ works. As $\left[\gamma_{0}, \rightarrow\right)_{\varrho}$ is uncountable, there are only countably many points from $\operatorname{Isol}\left(\omega_{1}\right) \varrho$-below $\gamma_{0}$, so there exists $\gamma_{1} \in \operatorname{Isol}\left(\omega_{1}\right) \cap \operatorname{int}_{\varrho} J \cap\left(\gamma_{0}, \rightarrow\right)_{\varrho}$. The $\varrho$-interval $\left[\gamma_{1}, \rightarrow\right)_{\varrho}$ is uncountable by our assumption, so the construction goes on up to $\omega_{1}$. (Claim 3 is used at limit steps.)

If Case 2 holds, then since $\operatorname{Isol}\left(\omega_{1}\right)$ is dense in $X$, we may assume that $a, b \in \operatorname{Isol}\left(\omega_{1}\right)$. Again, by recursion (and using Claim 3) one can construct uncountable $\varrho$-intervals $J_{\alpha}=\left[a_{\alpha}, b_{\alpha}\right]_{\varrho}$ such that int $J_{\alpha} \supset J_{\alpha^{\prime}}$ if $\alpha<\alpha^{\prime}<\omega_{1}$, so both $\left\{a_{\alpha}: \alpha \in \omega_{1}\right\}$ and $\left\{b_{\alpha}: \alpha \in \omega_{1}\right\}$ are uncountable subsets of Isol $\left(\omega_{1}\right)$ whose existence contradicts Claim 1 .

This finishes the proof of Fact 4 and, hence, of Theorem 4.6.

In the next example, we show that the condition $h(X)<\omega$ is necessary in Theorem 3.5. Furthermore, we prove that an LCC-space with a continuous zero-selection is not necessarily normal, even if its height is 2 .

$\left({ }^{3}\right) \operatorname{int}_{\varrho} J$ consists of the points which are not in the $\varrho$-boundary of $J$. 
THEOREM 4.7. Assume $\diamond$. There exist a subset $S$ of $\omega_{1}$ and a topology $\tau$ on $S$ finer than the relative topology of $\omega_{1}$ such that $X=(S, \tau)$ is a collectionwise normal LCC-space of height $\omega$ with a continuous zero-selection, but $X$ is not suborderable. The union $Y$ of the first two levels of $X$ fails to be normal.

Proof. Take a $\diamond$-sequence $\left\{A_{\alpha}: \alpha<\omega_{1}\right\}$. Using Proposition 4.4, define inductively $H^{0}=\operatorname{Isol}\left(\omega_{1}\right), H^{n+1}=I\left(H^{n}\right)$ for every $n \in \omega$ and $S=$ $\bigcup_{n=0}^{\infty} H^{n}$. One can apply the definition of $I\left(\right.$ ) to prove that the sets $H^{n}$ are mutually disjoint. We shall construct a scattered topology on $S$ for which $H^{n}$ is the $n$-level. By induction on $n$, we define a base $\mathcal{B}(\alpha)$ for each point $\alpha \in H^{n}$ consisting of topped compact open neighborhoods.

- If $\alpha \in H^{0}$, then $\mathcal{B}(\alpha)=\{\{\alpha\}\}$.

- Suppose we have already defined $\mathcal{B}(\alpha)$ for all points from $\bigcup_{k \leq n} H^{k}$. Let $\alpha \in H^{n+1}=I\left(H^{n}\right)$. Then $H^{n} \supseteq A_{\alpha}$ and $\sup A_{\alpha}=\alpha$. Choose a sequence $\left\{\sigma^{\alpha}(k): k \in \omega\right\} \subseteq A_{\alpha}$ such that $\sigma^{\alpha}(k) \nearrow \alpha$ and $\sigma^{\alpha}(2 k+1)$ is the successor of $\sigma^{\alpha}(2 k)$ in the order of $A_{\alpha}$. For each $k \geq 1$, take a compact open set $W_{k} \in \mathcal{B}\left(\sigma^{\alpha}(k)\right)$ such that $W_{k} \subseteq\left(\sigma^{\alpha}(k-1), \sigma^{\alpha}(k)\right]$. Put $V_{k}(\alpha)=\{\alpha\} \cup \bigcup_{l \geq k} W_{l}$. The local base at $\alpha$ is defined as $\mathcal{B}(\alpha)=\left\{V_{k}(\alpha)\right.$ : $k \geq 1\}$.

Let $\tau$ be the topology on $S$ with the base $\bigcup_{\alpha<\omega_{1}} \mathcal{B}(\alpha)$. Then $\tau$ is finer than the relative topology of the ordinal space $\omega_{1}, X=(S, \tau)$ is an LCC-space with a continuous zero-selection (apply Lemma 2.2), and its levels are the sets $H^{n}$. A direct verification shows that the statement of Fact 3 in the proof of Theorem 4.6 remains valid for $X$. A useful fact for transferring Fact 3 is that for each uncountable $C \subseteq S, \bar{C}^{\tau} \cap H^{n}$ is uncountable for all but finitely many $n \in \omega$.

Now we prove that $X$ is collectionwise normal. Let $\left\{F_{\delta}: \delta \in D\right\}$ be a discrete family of closed subsets of $X$. We may distinguish three cases:

- There exists $\gamma<\omega_{1}$ such that $F_{\delta} \subseteq \gamma+1$ for every $\delta \in D$. Since the countable subspace $X_{\gamma}=\{\alpha \in S: \alpha \leq \gamma\}$ is clopen and Lindelöf, there exists a disjoint family $\left\{V_{\delta}\right\}_{\delta \in D}$ of open subsets of $X$ such that $F_{\delta} \subseteq V_{\delta}$ for every $\delta \in D[12,5.1 .17-18]$.

- There exists $\delta_{0} \in D$ such that $F_{\delta_{0}}$ is unbounded in $\omega_{1}$. Put $G=$ $\bigcup_{\delta \neq \delta_{0}} F_{\delta}$. By Fact 3, $G$ is bounded. Arguing as above, we can find a disjoint family $\left\{V_{\delta}\right\}_{\delta \in D}$ of open subsets of $X$ such that $F_{\delta_{0}} \cap X_{\gamma} \subseteq V_{\delta_{0}}$ and $F_{\delta} \subseteq V_{\delta}$ whenever $\delta \neq \delta_{0}$. The required disjoint family of open sets is $\left\{V_{\delta}: \delta \in\right.$ $\left.D \backslash\left\{\delta_{0}\right\}\right\} \cup\left\{V_{\delta_{0}} \cup\left(X \backslash X_{\gamma}\right)\right\}$.

- Every $F_{\delta}$ is bounded, but $\bigcup_{\delta \in D} F_{\delta}$ is unbounded. It is easy to find disjoint subsets $D_{1}$ and $D_{2}$ of $D$ such that $G_{i}=\bigcup_{\delta \in D_{i}} F_{\delta}$ is closed and unbounded for $i \in\{1,2\}$, thus contradicting Fact 3 . 
It remains to show that the open subspace $Y=H^{0} \cup H^{1}$ of $X$ is not normal (a suborderable space is hereditarily normal). Notice that $H^{1}$ is closed and discrete in $Y$ and it is stationary in $\omega_{1}$. Let $G$ be an unbounded non-stationary subset of $H^{1}$. Then $G$ and $H^{1} \backslash G$ are disjoint closed subsets of $Y$. If $\Omega$ is an open set containing $G$, let $B=\Omega \cap H^{0}$. Then $B$ is an uncountable subset of $\operatorname{Isol}\left(\omega_{1}\right)$, the set $A(B)$ is stationary and $A(B) \subseteq H^{1}$. Consequently, $A(B) \backslash G$ is a non-empty subset of $H^{1} \backslash G$. If $\alpha \in A(B)$, then $\alpha \in \bar{A}_{\alpha}^{\tau} \subseteq \bar{B}^{\tau} \subseteq \bar{\Omega}^{\tau}$. Therefore, $\left(H^{1} \backslash G\right) \cap \bar{\Omega}^{\tau} \neq \emptyset$, and $Y$ cannot be normal.

5. Open problems. By van Douwen's result in [9], every countably compact space with a continuous weak selection is sequentially compact. The following problem is an attempt to find an analog of this fact for pseudocompact spaces.

Problem 5.1. Is a pseudocompact space $X$ with a continuous weak selection necessarily sequentially pseudocompact or does it belong to the Frolik class $\mathcal{B}$ ? $\left(^{4}\right)$

In view of Corollary 1.4 and Proposition 1.9, the above problem is equivalent to the following one:

Problem 5.2. Is every pseudocompact space $X$ with a continuous weak selection suborderable?

Counterexamples in Section 4 have been constructed under $\diamond$. We do not know whether similar spaces exist in ZFC.

Problem 5.3. Does there exist in ZFC a space as in Theorem 4.6, i.e., a locally compact locally countable monotonically (or hereditarily collectionwise) normal space with a continuous zero-selection which is not a subspace of ordinals? $\left({ }^{5}\right)$

Recall that by Theorem 3.1 and Lemma 3.2, a space as in Problem 5.3 must have uncountable height. Problem 5.3 remains open, even if the requirement of local countability is omitted. If, however, local compactness is omitted, then it is easy to find an example in ZFC: see [4]. In addition, once we drop both local compactness and local countability, a space like that in Theorem 4.7 can be constructed in ZFC using an appropriate ladder system. This indicates that the spaces with a continuous zero-selection are very

$\left({ }^{4}\right)$ Garcia-Ferreira and Sanchis have recently answered this problem in the affirmative: a continuous weak selection implies sequential pseudocompactness for pseudocompact spaces.

$\left({ }^{5}\right)$ It will be clarified in the forthcoming paper [4] that replacing a subspace of ordinals by suborderable, one obtains the same problem. 
far from being orderable. Nevertheless, we dare to formulate the following conjecture (cf. Lemma 2.2):

ConjeCture 5.4. Let $X$ be a completely regular space with a continuous zero-selection. Then there is a continuous 1-1 map of $X$ to an ordinal space.

Acknowledgements. We thank the referee for a number of comments which helped us to substantially improve the presentation of the paper. We are also indebted to our highly esteemed colleagues, including G. De Marco, V. Gutev, P. Szeptycki and S. Todorčević, for many inspiring discussions.

\section{References}

[1] A. V. Arkhangel'skiǔ, Classes of topological groups, Russian Math. Surveys 36 (1981), 151-174; Russian original in: Uspekhi Mat. Nauk 36 (1981), no. 3, 127-146.

[2] -, Continuous images of Lindelöf $\Sigma$-groups, Soviet Math. Dokl. 36 (1988), 169-173.

[3] G. Artico and U. Marconi, Selections and topologically well-ordered spaces, Topology Appl. 115 (2001), 299-303.

[4] G. Artico, U. Marconi and J. Pelant, On zero-selections, in preparation.

[5] J. L. Blasco and M. Sanchis, On the product of two $b_{f}$-spaces, Acta Math. Acad. Sci. Hungar. 62 (1993), 111-118.

[6] E. Čech, Topological Spaces, revised by Z. Frolík and M. Katětov, Czechoslovak Acad. Sci., Prague, 1966.

[7] W. W. Comfort and K. A. Ross, Pseudocompactness and uniform continuity in topological groups, Pacific J. Math. 16 (1966), 483-496.

[8] W. W. Comfort and F. J. Trigos-Arrieta, Locally pseudocompact topological groups, Topology Appl. 62 (1995), 263-280.

[9] E. K. van Douwen, Mappings from hyperspaces and convergent sequences, ibid. 34 (1990), 35-45.

[10] S. Eilenberg, Ordered topological spaces, Amer. J. Math. 63 (1941), 39-45.

[11] P. C. Eklof and A. H. Mekler, Almost Free Modules: Set-Theoretic Methods, Elsevier, Amsterdam, 1990.

[12] R. Engelking, General Topology, Heldermann, Berlin, 1989.

[13] Z. Frolík, The topological product of two pseudocompact spaces, Czechoslovak Math. J. 10 (85) (1960), 339-349.

[14] S. Fujii and T. Nogura, Characterizations of compact ordinal spaces via continuous selections, Topology Appl. 20 (1997), 1-5.

[15] L. Gillman and M. Jerison, Rings of Continuous Functions, Van Nostrand, New York, 1960.

[16] I. Glicksberg, Stone-Čech compactifications of products, Trans. Amer. Math. Soc. 90 (1959), 369-382.

[17] M. I. Graev, Free topological groups, in: Topology and Topological Algebra, Transl. Ser. 1, Vol. 8, Amer. Math. Soc., 1962, 305-364; Russian original in: Izv. Akad. Nauk SSSR Ser. Mat. 12 (1948), 279-324.

[18] J. de Groot, Non-archimedean metrics in topology, Proc. Amer. Math. Soc. 7 (1956), 948-953. 
[19] G. Gruenhage, Generalized metric spaces, in [24], 423-501.

[20] V. G. Gutev, Fell-continuous selections and topologically well-orderable spaces II, in: Proc. Ninth Topological Symposium (Prague, 2001), P. Simon (ed.), Topology Atlas, 2002, 147-153; arXiv:math.GN/0204129.

[21] V. G. Gutev and T. Nogura, Fell continuous selections and topologically well-orderable spaces, preprint, 1999, 8 pp.

[22] H. Herrlich, Ordnungsfähigkeit total-diskontinuierlicher Räume, Math. Ann. 159 (1965), 77-80.

[23] K. Kunen, Set Theory. An Introduction to Independence Proofs, North-Holland, Amsterdam, 1980.

[24] K. Kunen and J. E. Vaughan (eds.), Handbook of Set-Theoretic Topology, Elsevier, Amsterdam, 1984.

[25] D. Kurepa, Sur l'écart abstrait, Glas. Mat. Fiz. Astr. 11 (1956), 105-134.

[26] V. Kuz'minov, On a hypothesis of P. S. Aleksandrov in the theory of topological groups, Dokl. Akad. Nauk SSSR 125 (1959), 727-729 (in Russian).

[27] L. F. McAuley, A note on complete collectionwise normality and paracompactness, Proc. Amer. Math. Soc. 9 (1958), 796-799.

[28] E. Michael, Topologies on spaces of subsets, Trans. Amer. Math. Soc. 71 (1951), $152-182$.

[29] J. van Mill and E. Wattel, Selections and orderability, Proc. Amer. Math. Soc. 83 (1981), 601-605.

[30] - - - Orderability from selections: another solution to the orderability problem, Fund. Math. 121 (1984), 219-229.

[31] T. Nogura and D. Shakhmatov, Characterizations of intervals via continuous selections, Rend. Circ. Mat. Palermo 46 (1997), 317-328.

[32] P. J. Nyikos and H.-C. Reichel, Topologically orderable groups, Gen. Topology Appl. 5 (1975), 195-204.

[33] S. Purisch, On the orderability of Stone- Čech compactifications, Proc. Amer. Math. Soc. 41 (1973), 55-56.

[34] - Scattered compactifications and the orderability of scattered spaces II, ibid. 95 (1985), 636-640.

[35] M. E. Rudin and S. Watson, Countable products of scattered paracompact spaces, Proc. Amer. Math. Soc. 89 (1983), 551-552.

[36] Z. Semadeni, Banach Spaces of Continuous Functions, PWN-Polish Sci. Publ., Warszawa, 1971.

[37] R. Telgársky, Total paracompactness and paracompact dispersed spaces, Bull. Acad. Polon. Sci. Sér. Sci. Math. Astronom. Phys. 16 (1968), 567-572.

[38] M. G. Tkachenko, Compactness type properties in topological groups, Czechoslovak Math. J. 38 (113) (1988), 324-341.

[39] F. J. Trigos-Arrieta, Products of locally pseudocompact topological groups, in: Papers on General Topology and Applications (Brookville, NY, 1990), Ann. New York Acad. Sci. 659, New York Acad. Sci., 1992, 182-187.

[40] V. V. Uspenskiǔ, Why compact groups are dyadic, in: General Topology and its Relations to Modern Analysis and Algebra, VI (Prague, 1986), Z Frolík (ed.), Heldermann, Berlin, 1988, 601-610.

[41] J. E. Vaughan, Countably compact and sequentially compact spaces, in [24], 569-602.

[42] -, On Frolik's characterization of class P, Czechoslovak Math. J. 44 (119) (1994), 1-6. 
[43] M. Venkataraman, M. Rajagopalan and T. Soundararajan, Orderable topological spaces, Gen. Topology Appl. 2 (1972), 1-10.

Dipartimento di Matematica Pura e Applicata via Belzoni 7

I-35131 Padova, Italy

E-mail: umarconi@math.unipd.it

Departamento de Matemáticas

Universidad Autónoma Metropolitana

Av. San Rafael Atlixco \# 186, Col. Vicentina

C.P. 09340 Iztapalapa

México, D.F., México

E-mail:mich@xanum.uam.mx
Mathematical Institute Academy of Sciences of Czech Republic Žitná 25

11567 Praha 1, Czech Republic

E-mail: pelant@beba.cesnet.cz

Received 31 May 2000;

in revised form 15 August 2001 and 6 May 2002 